\title{
STABLE QUOTIENTS AND THE HOLOMORPHIC ANOMALY EQUATION
}

\author{
HYENHO LHO AND RAHUL PANDHARIPANDE
}

\begin{abstract}
We study the fundamental relationship between stable quotient invariants and the B-model for local $\mathbb{P}^{2}$ in all genera. Our main result is a direct geometric proof of the holomorphic anomaly equation in the precise form predicted by B-model physics. The method yields new holomorphic anomaly equations for an infinite class of twisted theories on projective spaces.

An example of such a twisted theory is the formal quintic defined by a hyperplane section of $\mathbb{P}^{4}$ in all genera via the Euler class of a complex. The formal quintic theory is found to satisfy the holomorphic anomaly equations conjectured for the true quintic theory. Therefore, the formal quintic theory and the true quintic theory should be related by transformations which respect the holomorphic anomaly equations.
\end{abstract}

\section{Contents}

0 . Introduction

1. Stable quotients 11

2. Localization graphs 14

3. Basic correlators 16

4. Higher genus series on $\bar{M}_{g, n}$

5. Higher genus series on $K \mathbb{P}^{2}$

6. Vertices, edges, and legs 35

7. Holomorphic anomaly for $K \mathbb{P}^{2}$

8. Holomorphic anomaly for $K \mathbb{P}^{2}$ with insertions 47

References 51

\section{INTRODUCTION}

0.1. GW/SQ. To the A-model Gromov-Witten theory of a CalabiYau 3-fold $X$ is conjecturally associated the B-model theory of a mirror Calabi-Yau 3-fold $Y$. At the genus 0 level, much is known about the geometry underlying the B-model: Hodge theory, period integrals, and

Date: March 2018. 
the linear sigma model. In higher genus, mathematical techniques have been less successful. In toric Calabi-Yau geometries, the topological recursion of Eynard and Orantin provides a link to the B-model [5, 16]. Another mathematical approach to the B-model (without the toric hypothesis) has been proposed by Costello and Li [14] following paths suggested by string theory. A very different mathematical view of the geometry of B-model invariants is pursued here.

Let $X_{5} \subset \mathbb{P}^{4}$ be a (nonsingular) quintic Calabi-Yau 3-fold. The moduli space of stable maps to the quintic of genus $g$ and degree $d$,

$$
\bar{M}_{g}\left(X_{5}, d\right) \subset \bar{M}_{g}\left(\mathbb{P}^{4}, d\right),
$$

has virtual dimension 0. The Gromov-Witten invariants ${ }^{1}$.

$$
N_{g, d}^{\mathrm{GW}}=\langle 1\rangle_{g, d}^{\mathrm{GW}}=\int_{\left[\bar{M}_{g}\left(X_{5}, d\right)\right]^{v i r}} 1,
$$

have been studied for more than 20 years, see [15, 18, 25] for an introduction to the subject.

The theory of stable quotients developed in [29] was partially inspired by the question of finding a geometric approach to a higher genus linear sigma model. The moduli space of stable quotients for the quintic,

$$
\bar{Q}_{g}\left(X_{5}, d\right) \subset \bar{Q}_{g}\left(\mathbb{P}^{4}, d\right)
$$

was defined in [29, Section 9], and questions about the associated integral theory,

$$
N_{g, d}^{\mathrm{SQ}}=\langle 1\rangle_{g, d}^{\mathrm{SQ}}=\int_{\left.\left[\bar{Q}_{g}\left(X_{5}, d\right)\right]\right]^{v i r}} 1
$$

were posed.

The existence of a natural obstruction theory on $\bar{Q}_{g}\left(X_{5}, d\right)$ and a virtual fundamental class $\left[\bar{Q}_{g}\left(X_{5}, d\right)\right]^{\text {vir }}$ is easily seen ${ }^{2}$ in genus 0 and 1 . A proposal in higher genus for the obstruction theory and virtual class was made in [29] and was carried out in significantly greater generality in the setting of quasimaps in [11. In genus 0 and 1, the integral theory (2) was calculated in [13] and [23] respectively. The answers on the stable quotient side exactly match the string theoretic B-model for the quintic in genus 0 and 1.

\footnotetext{
${ }^{1}$ In degree 0 , the moduli spaces of maps and stable quotients are both empty for genus 0 and 1 , so the invariants in these cases vanish.

${ }^{2}$ For stability, marked points are required in genus 0 and positive degree is required in genus 1.
} 
A relationship in every genus between the Gromov-Witten and stable quotient invariants of the quintic has been recently proven by CiocanFontanine and $\operatorname{Kim}[9]]^{3}$ Let $H \in H^{2}\left(X_{5}, \mathbb{Z}\right)$ be the hyperplane class of the quintic, and let

$$
\begin{gathered}
\mathcal{F}_{g, n}^{\mathrm{GW}}(Q)=\langle\underbrace{H, \ldots, H}_{n}\rangle_{g, n}^{\mathrm{GW}}=\sum_{d=0}^{\infty} Q^{d} \int_{\left[\bar{M}_{g, n}\left(X_{5}, d\right)\right]} \prod_{i=1}^{n} \mathrm{ev}_{i}^{*}(H), \\
\mathcal{F}_{g, n}^{\mathrm{SQ}}(q)=\langle\underbrace{H, \ldots, H}_{n}\rangle_{g, n}^{\mathrm{SQ}}=\sum_{d=0}^{\infty} q^{d} \int_{\left[\bar{Q}_{g, n}\left(X_{5}, d\right)\right]} \prod_{i=1}^{n} \operatorname{ev}_{i}^{*}(H)
\end{gathered}
$$

be the Gromov-Witten and stable quotient series respectively (involving the pointed moduli spaces and the evaluation morphisms at the markings). Let

$I_{0}^{\mathrm{Q}}(q)=\sum_{d=0}^{\infty} q^{d} \frac{(5 d) !}{(d !)^{5}}, \quad I_{1}^{\mathrm{Q}}(q)=\log (q) I_{0}^{\mathrm{Q}}(q)+5 \sum_{d=1}^{\infty} q^{d} \frac{(5 d) !}{(d !)^{5}}\left(\sum_{r=d+1}^{5 d} \frac{1}{r}\right)$.

The mirror map is defined by

$$
Q(q)=\exp \left(\frac{I_{1}^{\mathrm{Q}}(q)}{I_{0}^{\mathrm{Q}}(q)}\right)=q \cdot \exp \left(\frac{5 \sum_{d=1}^{\infty} q^{d} \frac{(5 d) !}{(d !)^{5}}\left(\sum_{r=d+1}^{5 d} \frac{1}{r}\right)}{\sum_{d=0}^{\infty} q^{d} \frac{(5 d) !}{(d !)^{5}}}\right) .
$$

The relationship between the Gromov-Witten and stable quotient invariants of the quintic in case

$$
2 g-2+n>0
$$

is given by the following result [9]:

$$
\mathcal{F}_{g, n}^{\mathrm{GW}}(Q(q))=I_{0}^{\mathrm{Q}}(q)^{2 g-2+n} \cdot \mathcal{F}_{g, n}^{\mathrm{SQ}}(q) .
$$

The transformation (3) shows the stable quotient theory matches the string theoretic B-model series for the quintic $X_{5}$.

Based on the above investigation of the quintic, a fundamental relationship between the stable quotient invariants and the B-model for all local and complete intersection Calabi-Yau 3-folds $X$ is natural to propose: the stable quotient invariants of $X$ exactly equal the B-model invariants of the mirror $Y$.

\footnotetext{
${ }^{3} \mathrm{~A}$ second proof (in most cases) can be found in [12].
} 
0.2. Holomorphic anomaly. The (conjectural) holomorphic anomaly equation is a beautiful property of the string theoretic B-model series which has been used effectively since [4. Since the stable quotients invariants provide a geometric proposal for the B-model series, we should look for the geometry of the holomorphic anomaly equation in the moduli space of stable quotients.

Since the stable quotients invariant for the quintic are difficult ${ }^{4}$ to approach in $g \geq 2$, we study here instead the stable quotient invariants in all genera for local $\mathbb{P}^{2}$ - the toric Calabi-Yau 3-fold given by the total space $K \mathbb{P}^{2}$ of the canonical bundle over $\mathbb{P}^{2}$. Our main result is a direct proof of the holomorphic anomaly equations in the precise form predicted by B-model physics for local $\mathbb{P}^{2}$.

The holomorphic anomaly equation can be proven for toric CalabiYau 3-folds by the topological recursion of Eynard and Orantin [16] together with the remodelling conjecture [5] proven recently in [17]. The path of [5, 16, 17] to the holomorphic anomaly equation for toric CalabiYau 3-folds involves explicit manipulation of the Gromov-Witten partition function (which can be computed by now in several different ways in toric Calabi-Yau cases [1, 30]). Our approach involves the geometry of the moduli space of stable quotients - the derivation of the holomorphic anomaly equation takes place on the stable quotient side.

The new perspective yields new holomorphic anomaly equations for an infinite class of twisted theories on projective spaces. Some of these are related to toric Calabi-Yau geometries (such as local $\mathbb{P}^{1} \times \mathbb{P}^{1}$ ), but most are not. The holomorphic anomaly equations for these theories have never been considered before.

A particular twisted theory on $\mathbb{P}^{4}$ is related to the quintic 3 -fold. Let the algebraic torus

$$
\mathrm{T}_{5}=\left(\mathbb{C}^{*}\right)^{5}
$$

act with the standard linearization on $\mathbb{P}^{4}$ with weights $\lambda_{0}, \ldots, \lambda_{4}$ on the vector space $H^{0}\left(\mathbb{P}^{4}, \mathcal{O}_{\mathbb{P}^{4}}(1)\right)$. Let

$$
\mathrm{C} \rightarrow \bar{M}_{g}\left(\mathbb{P}^{4}, d\right), \quad f: \mathrm{C} \rightarrow \mathbb{P}^{4}, \quad \mathrm{~S}=f^{*} \mathcal{O}_{\mathbb{P}^{4}}(-1) \rightarrow \mathrm{C}
$$

be the universal curve, the universal map, and the universal bundle over the moduli space of stable maps - all equipped with canonical

\footnotetext{
${ }^{4}$ There are very few mathematical derivations of the holomorphic anomaly equation in higher genus for compact Calabi-Yau 3-folds. A genus 2 result for the Enriques Calabi-Yau can be found in [31].
} 
$\mathrm{T}_{5}$-actions. We define the formal quintic invariants by

$$
\tilde{N}_{g, d}^{\mathrm{GW}}=\int_{\left.\left[\bar{M}_{g}\left(\mathbb{P}^{4}, d\right)\right]\right]^{v i r}} e\left(R \pi_{*}\left(\mathrm{~S}^{-5}\right)\right),
$$

where $e\left(R \pi_{*}\left(\mathrm{~S}^{-5}\right)\right)$ is the equivariant Euler class (defined after localization). The integral (5) is homogeneous of degree 0 in localized equivariant cohomology,

$$
\int_{\left[\bar{M}_{g}\left(\mathbb{P}^{4}, d\right)\right]^{v i r}} e\left(R \pi_{*}\left(\mathrm{~S}^{-5}\right)\right) \in \mathbb{Q}\left(\lambda_{0}, \ldots, \lambda_{4}\right),
$$

and defines a rational number $\widetilde{N}_{g, d}^{\mathrm{GW}} \in \mathbb{Q}$ after the specialization

$$
\lambda_{i}=\zeta^{i} \lambda_{0}
$$

for a primitive fifth root of unity $\zeta^{5}=1$.

A main result in the sequel [27] proves that the holomorphic anomaly equations conjectured for the true quintic theory (1) are satisfied by the formal quintic theory (5). In particular, the formal quintic theory and the true quintic theory should be related by transformations which respect the holomorphic anomaly equations.

0.3. Twisted theories on $\mathbb{P}^{m}$. Twisted theories associated to $\mathbb{P}^{m}$ can be constructed as follows. Let the algebraic torus

$$
\mathrm{T}_{m+1}=\left(\mathbb{C}^{*}\right)^{m+1}
$$

act with the standard linearization on $\mathbb{P}^{m}$ with weights $\lambda_{0}, \ldots, \lambda_{m}$ on the vector space $H^{0}\left(\mathbb{P}^{m}, \mathcal{O}_{\mathbb{P}^{m}}(1)\right)$.

Let $\bar{M}_{g}\left(\mathbb{P}^{m}, d\right)$ be the moduli space of stable maps to $\mathbb{P}^{m}$ equipped with the canonical $\mathrm{T}_{m+1}$-action, and let

$$
\mathrm{C} \rightarrow \bar{M}_{g}\left(\mathbb{P}^{m}, d\right), \quad f: \mathrm{C} \rightarrow \mathbb{P}^{m}, \quad \mathrm{~S}=f^{*} \mathcal{O}_{\mathbb{P}^{m}}(-1) \rightarrow \mathrm{C}
$$

be the standard universal structures. Let

$$
\mathrm{a}=\left(a_{1}, \ldots, a_{r}\right), \quad \mathrm{b}=\left(b_{1}, \ldots, b_{s}\right)
$$

be vectors of positive integers satisfying the conditions

$$
\sum_{i=1}^{r} a_{i}-\sum_{j=1}^{s} b_{j}=m+1 \quad \text { and } \quad m-r+s=3 .
$$

The first is the Calabi-Yau condition and the second is the dimension 3 condition.

\footnotetext{
${ }^{5}$ The negative exponent denotes the dual: $S$ is a line bundle and $S^{-5}=\left(S^{\star}\right)^{\otimes 5}$.
} 
The Gromov-Witten invariants of the $(\mathrm{a}, \mathrm{b})$-twisted geometry of $\mathbb{P}^{m}$ are defined via the equivariant integrals

$$
\widetilde{N}_{g, d}^{\mathrm{GW}}=\int_{\left[\bar{M}_{g}\left(\mathbb{P}^{m}, d\right)\right]^{v i r}} \prod_{i=1}^{r} e\left(R \pi_{*} \mathrm{~S}^{-a_{i}}\right) \prod_{j=1}^{s} e\left(-R \pi_{*} \mathrm{~S}^{b_{j}}\right) .
$$

The integral (6) is homogeneous of degree 0 in localized equivariant cohomology and defines a rational number

$$
\widetilde{N}_{g, d}^{\mathrm{GW}} \in \mathbb{Q}
$$

after the specialization

$$
\lambda_{i}=\zeta^{i} \lambda_{0}
$$

for a primitive $(m+1)$-root of unity $\zeta^{m+1}=1$.

The standard theory of local $\mathbb{P}^{2}$ theory is recovered in case

$$
m=2, \quad r=0, \quad b=(3) \text {. }
$$

We will see in 28] that the case

$$
m=3, \quad \mathrm{a}=(2), \quad \mathrm{b}=(2)
$$

is related to the local $\mathbb{P}^{1} \times \mathbb{P}^{1}$ geometry. The formal quintic theory arises in case

$$
m=4, \quad \mathrm{a}=(5), \quad s=0 .
$$

The stable quotient perspective of the paper yields holomorphic anomaly equations for all $(\mathrm{a}, \mathrm{b})$-twisted theories on $\mathbb{P}^{m}$ satisfying the Calabi-Yau and dimension 3 conditions. Our goal here is to present two of the most interesting cases.

0.4. Holomorphic anomaly for $K \mathbb{P}^{2}$. We state here the precise form of the holomorphic anomaly equations for local $\mathbb{P}^{2}$.

Let $H \in H^{2}\left(K \mathbb{P}^{2}, \mathbb{Z}\right)$ be the hyperplane class of the obtained from $\mathbb{P}^{2}$, and let

$$
\begin{gathered}
\mathcal{F}_{g, n}^{\mathrm{GW}}(Q)=\langle\underbrace{H, \ldots, H}_{n}\rangle_{g, n}^{\mathrm{GW}}=\sum_{d=0}^{\infty} Q^{d} \int_{\left.\left[\bar{M}_{g, n}\left(K \mathbb{P}^{2}, d\right)\right]\right]^{v i r}} \prod_{i=1}^{n} \mathrm{ev}_{i}^{*}(H), \\
\mathcal{F}_{g, n}^{\mathrm{SQ}}(q)=\langle\underbrace{H, \ldots, H}_{n}\rangle_{g, n}^{\mathrm{SQ}}=\sum_{d=0}^{\infty} q^{d} \int_{\left[\bar{Q}_{g, n}\left(K \mathbb{P}^{2}, d\right)\right]^{v i r}} \prod_{i=1}^{n} \mathrm{ev}_{i}^{*}(H)
\end{gathered}
$$

be the Gromov-Witten and stable quotient series respectively (involving the evaluation morphisms at the markings). The relationship between the Gromov-Witten and stable quotient invariants of $K \mathrm{P}^{2}$ in case $2 g-2+n>0$ is proven in [10]:

$$
\mathcal{F}_{g, n}^{\mathrm{GW}}(Q(q))=\mathcal{F}_{g, n}^{\mathrm{SQ}}(q)
$$


where $Q(q)$ is the mirror map,

$$
\begin{gathered}
I_{1}^{K \mathbb{P}^{2}}(q)=\log (q)+3 \sum_{d=1}^{\infty}(-q)^{d} \frac{(3 d-1) !}{(d !)^{3}} \\
Q(q)=\exp \left(I_{1}^{K \mathbb{P}^{2}}(q)\right)=q \cdot \exp \left(3 \sum_{d=1}^{\infty}(-q)^{d} \frac{(3 d-1) !}{(d !)^{3}}\right) .
\end{gathered}
$$

Again, the stable quotient theory matches the string theoretic B-model series for $K \mathbb{P}^{2}$.

In order to state the holomorphic anomaly equations, we require the following additional series in $q$.

$$
\begin{aligned}
L(q) & =(1+27 q)^{-\frac{1}{3}}=1-9 q+162 q^{2}+\ldots \\
C_{1}(q) & =q \frac{d}{d q} I_{1}^{K \mathbb{P}^{2}} \\
A_{2}(q) & =\frac{1}{L^{3}}\left(3 \frac{q \frac{d}{d q} C_{1}}{C_{1}}+1-\frac{L^{3}}{2}\right) .
\end{aligned}
$$

Let $T$ be the standard coordinate mirror to $t=\log (q)$,

$$
T=I_{1}^{K \mathbb{P}^{2}}(q) .
$$

Then $Q(q)=\exp (T)$ is the mirror map.

The ring $\mathbb{C}\left[L^{ \pm 1}\right]=\mathbb{C}\left[L, L^{-1}\right]$ will play basic role. Since

$$
q=\frac{1}{27}\left(L^{-3}-1\right)
$$

we have $\mathbb{C}[q] \subset \mathbb{C}\left[L^{ \pm 1}\right]$. For the holomorphic anomaly equation, consider the free polynomial rings in the variables $A_{2}$ and $C_{1}^{-1}$ over $\mathbb{C}\left[L^{ \pm 1}\right]$,

$$
\mathbb{C}\left[L^{ \pm 1}\right]\left[A_{2}\right], \quad \mathbb{C}\left[L^{ \pm 1}\right]\left[A_{2}, C_{1}^{-1}\right] .
$$

There are canonical maps

$$
\mathbb{C}\left[L^{ \pm 1}\right]\left[A_{2}\right] \rightarrow \mathbb{C}[[q]], \quad \mathbb{C}\left[L^{ \pm 1}\right]\left[A_{2}, C_{1}^{-1}\right] \rightarrow \mathbb{C}[[q]]
$$

given by assigning the above defined series $A_{2}(q)$ and $C_{1}^{-1}(q)$ to the variables $A_{2}$ and $C_{1}^{-1}$ respectively. We may therefore consider elements of the rings $(9)$ either as free polynomials in the variables $A_{2}$ and $C_{1}^{-1}$ or as series in $q$.

Let $F(q) \in \mathbb{Q}[[q]]$ be a series in $q$. When we write

$$
F(q) \in \mathbb{C}\left[L^{ \pm 1}\right]\left[A_{2}\right],
$$

we mean there is a canonical lift $F \in \mathbb{C}\left[L^{ \pm 1}\right]\left[A_{2}\right]$ for which

$$
F \mapsto F(q) \in \mathbb{C}[[q]]
$$


under the map (10). The symbol $F$ without the argument $q$ is the lift. The notation

$$
F(q) \in \mathbb{C}\left[L^{ \pm 1}\right]\left[A_{2}, C_{1}^{-1}\right]
$$

is parallel.

Theorem 1. For the stable quotient invariants of $K \mathbb{P}^{2}$,

(i) $\mathcal{F}_{g}^{S Q}(q) \in \mathbb{C}\left[L^{ \pm 1}\right]\left[A_{2}\right]$ for $g \geq 2$,

(ii) $\mathcal{F}_{g}^{\mathrm{SQ}}$ is of degree at most $3 g-3$ with respect to $A_{2}$,

(iii) $\frac{\partial^{k} \mathcal{F}_{g}^{\mathrm{SQ}}}{\partial T^{k}}(q) \in \mathbb{C}\left[L^{ \pm 1}\right]\left[A_{2}, C_{1}^{-1}\right]$ for $g \geq 1$ and $k \geq 1$,

(iv) $\frac{\partial^{k} \mathcal{F}_{g}^{\mathrm{SQ}}}{\partial T^{k}}$ is homogeneous of degree $k$ with respect to $C_{1}^{-1}$.

Here, $\mathcal{F}_{g}^{\mathrm{SQ}}=\mathcal{F}_{g, 0}^{\mathrm{SQ}}$.

Theorem 2. The holomorphic anomaly equations for the stable quotient invariants of $K \mathbb{P}^{2}$ hold for $g \geq 2$ :

$$
\frac{1}{C_{1}^{2}} \frac{\partial \mathcal{F}_{g}^{\mathrm{SQ}}}{\partial A_{2}}=\frac{1}{2} \sum_{i=1}^{g-1} \frac{\partial \mathcal{F}_{g-i}^{\mathrm{SQ}}}{\partial T} \frac{\partial \mathcal{F}_{i}^{\mathrm{SQ}}}{\partial T}+\frac{1}{2} \frac{\partial^{2} \mathcal{F}_{g-1}^{\mathrm{SQ}}}{\partial T^{2}}
$$

The derivative of $\mathcal{F}_{g}^{\mathrm{SQ}}$ (the lift) with respect to $A_{2}$ in the holomorphic anomaly equation of Theorem 2 is well-defined since

$$
\mathcal{F}_{g}^{\mathrm{SQ}} \in \mathbb{C}\left[L^{ \pm 1}\right]\left[A_{2}\right]
$$

by Theorem 1 part (i). By Theorem 1 parts (ii) and (iii),

$$
\frac{\partial \mathcal{F}_{g-i}^{\mathrm{SQ}}}{\partial T} \frac{\partial \mathcal{F}_{i}^{\mathrm{SQ}}}{\partial T}, \frac{\partial^{2} \mathcal{F}_{g-1}^{\mathrm{SQ}}}{\partial T^{2}} \in \mathbb{C}\left[L^{ \pm 1}\right]\left[A_{2}, C_{1}^{-1}\right]
$$

are both of degree 2 in $C_{1}^{-1}$. Hence, the holomorphic anomaly equation of Theorem 2 may be viewed as holding in $\mathbb{C}\left[L^{ \pm 1}\right]\left[A_{2}\right]$ since the factors of $C_{1}^{-1}$ on the left and right sides cancel. The holomorphic anomaly equations here for $K \mathbb{P}^{2}$ are exactly as presented in [2, (4.27)] via Bmodel physics.

Theorem 2 determines $\mathcal{F}_{g}^{\mathrm{SQ}} \in \mathbb{C}\left[L^{ \pm 1}\right]$ uniquely as a polynomial in $A_{2}$ up to a constant term in $\mathbb{C}\left[L^{ \pm 1}\right]$. In fact, the degree of the constant term can be bounded (as will be seen in the proof of Theorem 2). So Theorem 2 determines $\mathcal{F}_{g}^{\mathrm{SQ}}$ from the lower genus theory together with a finite amount of data.

0.5. Plan of the paper. After a review of the moduli space of stable quotients in Section 1 and the associated T-fixed point loci in Section 2 , the corresponding $I$-functions and vertex integrals are discussed in Sections 3 and 4 . The localization formula for $K \mathbb{P}^{2}$ in the precise form 
required for the holomorphic anomaly equation is presented in Sections 5 and 6. Theorems 1 and 2 are proven in Section 7 .

At the end of paper, in Section 8, we prove a new holomorphic anomaly equation for a stable quotient theory of $K \mathbb{P}^{2}$ with insertions parallel to (and inspired by) the recent work of Oberdieck and Pixton [32] on a cycle level holomorphic anomaly equation for the theory of an elliptic curve.

0.6. Holomorphic anomaly for the formal quintic. Let $g \geq 2$. The Gromov-Witten invariants of the formal quintic theory were defined in Section 0.2. The associated generating series is

$$
\widetilde{\mathcal{F}}_{g}^{\mathrm{GW}}(Q)=\sum_{d=0}^{\infty} \widetilde{N}_{g, d}^{\mathrm{GW}} Q^{d} \in \mathbb{C}[[Q]]
$$

We define the generating series of stable quotient invariants for formal quintic theory by the wall-crossing formula (3) for the true quintic theory,

$$
\widetilde{\mathcal{F}}_{g}^{\mathrm{GW}}(Q(q))=I_{0}^{\mathrm{Q}}(q)^{2 g-2} \cdot \widetilde{\mathcal{F}}_{g}^{\mathrm{SQ}}(q)
$$

with respect to the true quintic mirror map

$$
Q(q)=\exp \left(\frac{I_{1}^{\mathrm{Q}}(q)}{I_{0}^{\mathrm{Q}}(q)}\right)=q \cdot \exp \left(\frac{5 \sum_{d=1}^{\infty} q^{d} \frac{(5 d) !}{(d !)^{5}}\left(\sum_{r=d+1}^{5 d} \frac{1}{r}\right)}{\sum_{d=0}^{\infty} \frac{(5 d) !}{(d !)^{5}}}\right)
$$

Denote the B-model side of 11 by

$$
\widetilde{F}_{g}^{\mathrm{B}}(q)=I_{0}^{\mathrm{Q}}(q)^{2 g-2} \widetilde{\mathcal{F}}_{g}^{\mathrm{SQ}}(q) .
$$

In order to state the holomorphic anomaly equations, we require several series in $q$. First, let

$$
L(q)=\left(1-5^{5} q\right)^{-\frac{1}{5}}=1+625 q+117185 q^{2}+\ldots .
$$

Let $\mathrm{D}=q \frac{d}{d q}$, and let

$$
C_{0}(q)=I_{0}^{\mathrm{Q}}, \quad C_{1}(q)=\mathrm{D}\left(\frac{I_{1}^{\mathrm{Q}}}{I_{0}^{\mathrm{Q}}}\right)
$$


where $I_{0}$ and $I_{1}$ and the hypergeometric series appearing in the mirror map for the true quintic theory. We define

$$
\begin{aligned}
K_{2}(q)= & -\frac{1}{L^{5}} \frac{\mathrm{D} C_{0}}{C_{0}}, \\
A_{2}(q)= & \frac{1}{L^{5}}\left(-\frac{1}{5} \frac{\mathrm{D} C_{1}}{C_{1}}-\frac{2}{5} \frac{\mathrm{D} C_{0}}{C_{0}}-\frac{3}{25}\right), \\
A_{4}(q)= & \frac{1}{L^{10}}\left(-\frac{1}{25}\left(\frac{\mathrm{D} C_{0}}{C_{0}}\right)^{2}-\frac{1}{25}\left(\frac{\mathrm{D} C_{0}}{C_{0}}\right)\left(\frac{\mathrm{D} C_{1}}{C_{1}}\right)\right. \\
& \left.+\frac{1}{25} \mathrm{D}\left(\frac{\mathrm{D} C_{0}}{C_{0}}\right)+\frac{2}{25^{2}}\right), \\
A_{6}(q)= & \frac{1}{31250 L^{15}}\left(4+125 \mathrm{D}\left(\frac{\mathrm{D} C_{0}}{C_{0}}\right)+50\left(\frac{\mathrm{D} C_{0}}{C_{0}}\right)\left(1+10 \mathrm{D}\left(\frac{\mathrm{D} C_{0}}{C_{0}}\right)\right)\right. \\
& -5 L^{5}\left(1+10\left(\frac{\mathrm{D} C_{0}}{C_{0}}\right)+25\left(\frac{\mathrm{D} C_{0}}{C_{0}}\right)^{2}+25 \mathrm{D}\left(\frac{q \frac{d}{d q} C_{0}}{C_{0}}\right)\right) \\
& \left.+125 \mathrm{D}^{2}\left(\frac{\mathrm{D} C_{0}}{C_{0}}\right)-125\left(\frac{\mathrm{D} C_{0}}{C_{0}}\right)^{2}\left(\left(\frac{\mathrm{D} C_{1}}{C_{1}}\right)-1\right)\right) .
\end{aligned}
$$

Let $T$ be the standard coordinate mirror to $t=\log (q)$,

$$
T=\frac{I_{1}^{\mathrm{Q}}(q)}{I_{0}^{\mathrm{Q}}(q)} .
$$

Then $Q(q)=\exp (T)$ is the mirror map. Let

$$
\mathbb{C}\left[L^{ \pm 1}\right]\left[A_{2}, A_{4}, A_{6}, C_{0}^{ \pm 1}, C_{1}^{-1}, K_{2}\right]
$$

be the free polynomial ring over $\mathbb{C}\left[L^{ \pm 1}\right]$.

Theorem 3. For the series $\widetilde{\mathcal{F}}_{g}^{\mathrm{B}}$ associated to the formal quintic,

(i) $\widetilde{\mathcal{F}}_{g}^{\mathrm{B}}(q) \in \mathbb{C}\left[L^{ \pm 1}\right]\left[A_{2}, A_{4}, A_{6}, C_{0}^{ \pm 1}, C_{1}^{-1}, K_{2}\right]$ for $g \geq 2$,

(ii) $\frac{\partial^{k} \widetilde{\mathcal{F}}_{g}^{\mathrm{B}}}{\partial T^{k}}(q) \in \mathbb{C}\left[L^{ \pm 1}\right]\left[A_{2}, A_{4}, A_{6}, C_{0}^{ \pm 1}, C_{1}^{-1}, K_{2}\right]$ for $g \geq 1, k \geq 1$,

(iii) $\frac{\partial^{k} \widetilde{\mathcal{F}}_{g}^{\mathrm{B}}}{\partial T^{k}}$ is homogeneous with respect to $C_{1}^{-1}$ of degree $k$. 
Theorem 4. The holomorphic anomaly equations for the series $\widetilde{\mathcal{F}}_{g}^{\mathrm{B}}$ associated to the formal quintic hold for $g \geq 2$ :

$$
\begin{aligned}
\frac{1}{C_{0}^{2} C_{1}^{2}} \frac{\partial \widetilde{\mathcal{F}}_{g}^{\mathrm{B}}}{\partial A_{2}}-\frac{1}{5 C_{0}^{2} C_{1}^{2}} \frac{\partial \widetilde{\mathcal{F}}_{g}^{\mathrm{B}}}{\partial A_{4}} K_{2}+\frac{1}{50 C_{0}^{2} C_{1}^{2}} \frac{\partial \widetilde{\mathcal{F}}_{g}^{\mathrm{B}}}{\partial A_{6}} K_{2}^{2}= \\
\frac{1}{2} \sum_{i=1}^{g-1} \frac{\partial \widetilde{\mathcal{F}}_{g-i}^{\mathrm{B}}}{\partial T} \frac{\partial \widetilde{\mathcal{F}}_{i}^{\mathrm{B}}}{\partial T}+\frac{1}{2} \frac{\partial^{2} \widetilde{\mathcal{F}}_{g-1}^{\mathrm{B}}}{\partial T^{2}} .
\end{aligned}
$$

The equality of Theorem 4 holds in the ring

$$
\mathbb{C}\left[L^{ \pm 1}\right]\left[A_{2}, A_{4}, A_{6}, C_{0}^{ \pm 1}, C_{1}^{-1}, K_{2}\right]
$$

Theorem 4 exactly matches ${ }^{6}$ the conjectural holomorphic anomaly equation [2, (2.52)] for the true quintic theory $\mathcal{F}_{g}^{\mathrm{SQ}}$. Theorems 3 and 4 will be proven in [27].

0.7. Acknowledgments. We are very grateful to I. Ciocan-Fontanine, E. Clader, Y. Cooper, F. Janda, B. Kim, A. Klemm, Y.-P. Lee, A. Marian, M. Mariño, D. Maulik, D. Oprea, Y. Ruan, E. Scheidegger, Y. Toda, and A. Zinger for discussions over the years about the moduli space of stable quotients and the invariants of Calabi-Yau geometries.

R.P. was partially supported by SNF-200020162928, ERC-2012-AdG320368-MCSK, SwissMAP, and the Einstein Stiftung. H.L. was supported by the grant ERC-2012-AdG-320368-MCSK. The results here were presented by H.L. in genus 2 at Curves on surfaces and 3-folds at the Bernoulli center in Lausanne in June 2016 and in all genus at Moduli of curves, sheaves, and K3 surfaces at Humboldt University in Berlin in February 2017.

\section{Stable quotients}

1.1. Overview. We review here the basic definitions related to the moduli space of stable quotients following [29]. While the quasimap theory [11] is more general (and the quasimap terminology of [9, 23. will appear later in our proofs), only the original moduli spaces of stable quotients are used in the paper.

\footnotetext{
${ }^{6}$ Our functions $K_{2}$ and $A_{2 k}$ are normalized differently with respect to $C_{0}$ and $C_{1}$. The dictionary to exactly match the notation of [2, (2.52)] is to multiply our $K_{2}$ by $\left(C_{0} C_{1}\right)^{2}$ and our $A_{2 k}$ by $\left(C_{0} C_{1}\right)^{2 k}$.
} 
1.2. Stability. Let $\left(C, p_{1}, \ldots, p_{n}\right)$ be an $n$-pointed quasi-stable curve:

- $C$ is a reduced, connected, complete, scheme of dimension 1 with at worst nodal singularities,

- the markings $p_{i}$ are distinct and lie in the nonsingular locus $p_{i} \in C^{n s}$.

Let $q$ be a quotient of the rank $N$ trivial bundle $C$,

$$
\mathbb{C}^{N} \otimes \mathcal{O}_{C} \stackrel{q}{\longrightarrow} Q \longrightarrow 0
$$

If the quotient sheaf $Q$ is locally free at the nodes and markings of $C$, then $q$ is a quasi-stable quotient. Quasi-stability of $q$ implies the associated kernel,

$$
0 \longrightarrow S \longrightarrow \mathbb{C}^{N} \otimes \mathcal{O}_{C} \stackrel{q}{\longrightarrow} Q \longrightarrow 0
$$

is a locally free sheaf on $C$. Let $r$ denote the rank of $S$.

Let $\left(C, p_{1}, \ldots, p_{n}\right)$ be an $n$-pointed quasi-stable curve equipped with a quasi-stable quotient $q$. The data $\left(C, p_{1}, \ldots, p_{n}, q\right)$ determine a stable quotient if the $\mathbb{Q}$-line bundle

$$
\omega_{C}\left(p_{1}+\ldots+p_{n}\right) \otimes\left(\wedge^{r} S^{*}\right)^{\otimes \epsilon}
$$

is ample on $C$ for every strictly positive $\epsilon \in \mathbb{Q}$. Quotient stability implies

$$
2 g-2+n \geq 0
$$

where $g$ is the arithmetic genus of $C$.

Viewed in concrete terms, no amount of positivity of $S^{*}$ can stabilize a genus 0 component

$$
\mathbb{P}^{1} \stackrel{=}{P} \subset C
$$

unless $P$ contains at least 2 nodes or markings. If $P$ contains exactly 2 nodes or markings, then $S^{*}$ must have positive degree.

A stable quotient $(C, q)$ yields a rational map from the underlying curve $C$ to the Grassmannian $\mathbb{G}(r, N)$. We be will mainly interested in the projective space case here,

$$
\mathbb{G}(1, m+1)=\mathbb{P}^{m},
$$

but the definitions are uniform for all Grassmannian targets.

1.3. Isomorphism. Let $\left(C, p_{1}, \ldots, p_{n}\right)$ be an $n$-pointed curve. Two quasi-stable quotients

$$
\mathbb{C}^{N} \otimes \mathcal{O}_{C} \stackrel{q}{\longrightarrow} Q \longrightarrow 0, \quad \mathbb{C}^{N} \otimes \mathcal{O}_{C} \stackrel{q^{\prime}}{\longrightarrow} Q^{\prime} \longrightarrow 0
$$

on $\left(C, p_{1}, \ldots, p_{n}\right)$ are strongly isomorphic if the associated kernels

$$
S, S^{\prime} \subset \mathbb{C}^{N} \otimes \mathcal{O}_{C}
$$

are equal. 
An isomorphism of quasi-stable quotients

$$
\phi:\left(C, p_{1}, \ldots, p_{n}, q\right) \longrightarrow\left(C^{\prime}, p_{1}^{\prime}, \ldots, p_{n}^{\prime}, q^{\prime}\right)
$$

is an isomorphism of pointed curves

$$
\phi:\left(C, p_{1}, \ldots, p_{n}\right) \stackrel{\sim}{\longrightarrow}\left(C^{\prime}, p_{1}^{\prime}, \ldots, p_{n}^{\prime}\right)
$$

such that the quotients $q$ and $\phi^{*}\left(q^{\prime}\right)$ are strongly isomorphic. Quasistable quotients (13) on the same curve $\left(C, p_{1}, \ldots, p_{n}\right)$ may be isomorphic without being strongly isomorphic.

The following result is proven in [29] by Quot scheme methods from the perspective of geometry relative to a divisor.

Theorem A. The moduli space of stable quotients $\bar{Q}_{g, n}(\mathbb{G}(r, N), d)$ parameterizing the data

$$
\left(C, p_{1}, \ldots, p_{n}, 0 \longrightarrow S \longrightarrow \mathbb{C}^{N} \otimes \mathcal{O}_{C} \stackrel{q}{\longrightarrow} Q \longrightarrow 0\right),
$$

with $\operatorname{rank}(S)=r$ and $\operatorname{deg}(S)=-d$, is a separated and proper DeligneMumford stack of finite type over $\mathbb{C}$.

1.4. Structures. Over the moduli space of stable quotients, there is a universal $n$-pointed curve

$$
\pi: \mathcal{C} \rightarrow \bar{Q}_{g, n}(\mathbb{G}(r, N), d)
$$

with a universal quotient

$$
0 \longrightarrow \mathrm{S} \longrightarrow \mathbb{C}^{N} \otimes \mathcal{O}_{\mathcal{C}} \stackrel{q_{U}}{\longrightarrow} \mathrm{Q} \longrightarrow 0
$$

The subsheaf $\mathrm{S}$ is locally free on $\mathcal{C}$ because of the stability condition.

The moduli space $\bar{Q}_{g, n}(\mathbb{G}(r, N), d)$ is equipped with two basic types of maps. If $2 g-2>0$, then the stabilization of $C$ determines a map

$$
\nu: \bar{Q}_{g, n}(\mathbb{G}(r, N), d) \rightarrow \bar{M}_{g, n}
$$

by forgetting the quotient.

The general linear group $\mathbf{G L}_{N}(\mathbb{C})$ acts on $\bar{Q}_{g, n}(\mathbb{G}(r, N), d)$ via the standard action on $\mathbb{C}^{N} \otimes \mathcal{O}_{C}$. The structures $\pi, q_{U}, \nu$ and the evaluations maps are all $\mathbf{G L}_{N}(\mathbb{C})$-equivariant. We will be interested in the diagonal torus action,

$$
\mathrm{T}_{N+1} \subset \mathbf{G L}_{N}(\mathbb{C})
$$


1.5. Obstruction theory. The moduli of stable quotients maps to the Artin stack of pointed domain curves

$$
\nu^{A}: \bar{Q}_{g, n}(\mathbb{G}(r, N), d) \rightarrow \mathcal{M}_{g, n}
$$

The moduli of stable quotients with fixed underlying pointed curve $\left[C, p_{1}, \ldots, p_{n}\right] \in \mathcal{M}_{g, n}$ is simply an open set of the Quot scheme of $C$. The following result of [29, Section 3.2] is obtained from the standard deformation theory of the Quot scheme.

Theorem B. The deformation theory of the Quot scheme determines a 2-term obstruction theory on the moduli space $\bar{Q}_{g, n}(\mathbb{G}(r, N), d)$ relative to $\nu^{A}$ given by $\operatorname{RHom}(S, Q)$.

More concretely, for the stable quotient,

$$
0 \longrightarrow S \longrightarrow \mathbb{C}^{N} \otimes \mathcal{O}_{C} \stackrel{q}{\longrightarrow} Q \longrightarrow 0
$$

the deformation and obstruction spaces relative to $\nu^{A}$ are $\operatorname{Hom}(S, Q)$ and $\operatorname{Ext}^{1}(S, Q)$ respectively. Since $S$ is locally free, the higher obstructions

$$
\operatorname{Ext}^{k}(S, Q)=H^{k}\left(C, S^{*} \otimes Q\right)=0, \quad k>1
$$

vanish since $C$ is a curve. An absolute 2-term obstruction theory on the moduli space $\bar{Q}_{g, n}(\mathbb{G}(r, N), d)$ is obtained from Theorem B and the smoothness of $\mathcal{M}_{g, n}$, see [3, 22].

The $\mathbf{G L}_{N}(\mathbb{C})$-action lifts to the obstruction theory, and the resulting virtual class is defined in $\mathbf{G L}_{N}(\mathbb{C})$-equivariant cycle theory,

$$
\left[\bar{Q}_{g, n}(\mathbb{G}(r, N), d)\right]^{\mathrm{vir}} \in A_{*}^{\mathrm{GL}_{N}(\mathbb{C})}\left(\bar{Q}_{g, n}(\mathbb{G}(r, N), d)\right) .
$$

Via the restriction to the torus (15), we obtain

$$
\left[\bar{Q}_{g, n}(\mathbb{G}(r, N), d)\right]^{\mathrm{vir}} \in A_{*}^{\top_{N+1}}\left(\bar{Q}_{g, n}(\mathbb{G}(r, N), d)\right) .
$$

\section{LOCALIZATION GRAPHS}

2.1. Torus action. Let $\mathrm{T}=\left(\mathbb{C}^{*}\right)^{m+1}$ act diagonally on the vector space $\mathbb{C}^{m+1}$ with weights

$$
-\lambda_{0}, \ldots,-\lambda_{m}
$$

Denote the T-fixed points of the induced T-action on $\mathbb{P}^{m}$ by

$$
p_{0}, \ldots, p_{m} \text {. }
$$

The weights of $\mathrm{T}$ on the tangent space $T_{p_{j}}\left(\mathbb{P}^{m}\right)$ are

$$
\lambda_{j}-\lambda_{0}, \ldots, \widehat{\lambda}_{j}-\lambda_{j}, \ldots, \lambda_{j}-\lambda_{m} .
$$

There is an induced T-action on the moduli space $\bar{Q}_{g, n}\left(\mathbb{P}^{m}, d\right)$. The localization formula of [22] applied to the virtual fundamental class 
$\left[\bar{Q}_{g, n}\left(\mathbb{P}^{m}, d\right)\right]^{\text {vir }}$ will play a fundamental role our paper. The T-fixed loci are represented in terms of dual graphs, and the contributions of the T-fixed loci are given by tautological classes. The formulas here are standard, see [23, 29].

2.2. Graphs. Let the genus $g$ and the number of markings $n$ for the moduli space be in the stable range

$$
2 g-2+n>0 .
$$

We can organize the T-fixed loci of $\bar{Q}_{g, n}\left(\mathbb{P}^{m}, d\right)$ according to decorated graphs. A decorated graph $\Gamma \in \mathrm{G}_{g, n}\left(\mathbb{P}^{m}\right)$ consists of the data $(\mathrm{V}, \mathrm{E}, \mathrm{N}, \mathrm{g}, \mathrm{p})$ where

(i) $\mathrm{V}$ is the vertex set,

(ii) $\mathrm{E}$ is the edge set (including possible self-edges),

(iii) $\mathrm{N}:\{1,2, \ldots, n\} \rightarrow \mathrm{V}$ is the marking assignment,

(iv) $\mathrm{g}: \mathrm{V} \rightarrow \mathbb{Z}_{\geq 0}$ is a genus assignment satisfying

$$
g=\sum_{v \in V} \mathrm{~g}(v)+h^{1}(\Gamma)
$$

and for which $(\mathrm{V}, \mathrm{E}, \mathrm{N}, \mathrm{g})$ is stable graph 7 ,

$(\mathrm{v}) \mathrm{p}: \mathrm{V} \rightarrow\left(\mathbb{P}^{m}\right)^{\mathrm{T}}$ is an assignment of a T-fixed point $\mathrm{p}(v)$ to each vertex $v \in \mathrm{V}$.

The markings $\mathrm{L}=\{1, \ldots, n\}$ are often called legs.

To each decorated graph $\Gamma \in \mathrm{G}_{g, n}\left(\mathbb{P}^{m}\right)$, we associate the set of fixed loci of

$$
\sum_{d \geq 0}\left[\bar{Q}_{g, n}\left(\mathbb{P}^{m}, d\right)\right]^{\mathrm{vir}} q^{d}
$$

with elements described as follows:

(a) If $\left\{v_{i_{1}}, \ldots, v_{i_{k}}\right\}=\left\{v \mid \mathrm{p}(v)=p_{i}\right\}$, then $f^{-1}\left(p_{i}\right)$ is a disjoint union of connected stable curves of genera $\mathrm{g}\left(v_{i_{1}}\right), \ldots, \mathrm{g}\left(v_{i_{k}}\right)$ and finitely many points.

(b) There is a bijective correspondence between the connected components of $C \backslash D$ and the set of edges and legs of $\Gamma$ respecting vertex incidence where $C$ is domain curve and $D$ is union of all subcurves of $C$ which appear in (a).

We write the localization formula as

$$
\sum_{d \geq 0}\left[\bar{Q}_{g, n}\left(\mathbb{P}^{m}, d\right)\right]^{\mathrm{vir}} q^{d}=\sum_{\Gamma \in \mathrm{G}_{g, n}\left(\mathbb{P}^{m}\right)} \operatorname{Cont}_{\Gamma} .
$$

While $\mathrm{G}_{g, n}\left(\mathbb{P}^{m}\right)$ is a finite set, each contribution Cont $_{\Gamma}$ is a series in $q$ obtained from an infinite sum over all edge possibilities (b).

\footnotetext{
${ }^{7}$ Corresponding to a stratum of the moduli space of stable curves $\bar{M}_{g, n}$.
} 
2.3. Unstable graphs. The moduli spaces of stable quotients

$$
\bar{Q}_{0,2}\left(\mathbb{P}^{m}, d\right) \quad \text { and } \quad \bar{Q}_{1,0}\left(\mathbb{P}^{m}, d\right)
$$

for $d>0$ are the only ${ }^{8}$ cases where the pair $(g, n)$ does not satisfy the Deligne-Mumford stability condition (16).

An appropriate set of decorated graphs $\mathrm{G}_{0,2}\left(\mathbb{P}^{m}\right)$ is easily defined: The graphs $\Gamma \in \mathrm{G}_{0,2}\left(\mathbb{P}^{m}\right)$ all have 2 vertices connected by a single edge. Each vertex carries a marking. All of the conditions (i)-(v) of Section 2.2 are satisfied except for the stability of $(\mathrm{V}, \mathrm{E}, \mathrm{N}, \gamma)$. The localization formula holds,

$$
\sum_{d \geq 1}\left[\bar{Q}_{0,2}\left(\mathbb{P}^{m}, d\right)\right]^{\text {vir }} q^{d}=\sum_{\Gamma \in \mathrm{G}_{0,2}\left(\mathbb{P}^{m}\right)} \text { Cont }_{\Gamma},
$$

For $\bar{Q}_{1,0}\left(\mathbb{P}^{m}, d\right)$, the matter is more problematic — usually a marking is introduced to break the symmetry.

\section{BASIC CORRELATORS}

3.1. Overview. We review here basic generating series in $q$ which arise in the genus 0 theory of quasimap invariants. The series will play a fundamental role in the calculations of Sections 4 - 7 related to the holomorphic anomaly equation for $K \mathbb{P}^{2}$.

We fix a torus action $\mathrm{T}=\left(\mathbb{C}^{*}\right)^{3}$ on $\mathbb{P}^{2}$ with weight: ${ }^{9}$

$$
-\lambda_{0},-\lambda_{1},-\lambda_{2}
$$

on the vector space $\mathbb{C}^{3}$. The $\mathrm{T}$-weight on the fiber over $p_{i}$ of the canonical bundle

$$
\mathcal{O}_{\mathbb{P}^{2}}(-3) \rightarrow \mathbb{P}^{2}
$$

is $-3 \lambda_{i}$. The toric Calabi-Yau $K \mathbb{P}^{2}$ is the total space of (18).

Since the quasimap invariants are independent of $\lambda_{i}$, we are free to use the specialization

$$
\lambda_{1}=\zeta \lambda_{0}, \quad \lambda_{2}=\zeta^{2} \lambda_{0}
$$

where $\zeta$ is a primitive third root of unity. Of course, we then have

$$
\begin{aligned}
\lambda_{0}+\lambda_{1}+\lambda_{2} & =0, \\
\lambda_{0} \lambda_{1}+\lambda_{1} \lambda_{2}+\lambda_{2} \lambda_{0} & =0 .
\end{aligned}
$$

The specialization (19) will be imposed for our entire study of $K \mathbb{P}^{2}$.

\footnotetext{
${ }^{8}$ The moduli spaces $\bar{Q}_{0,0}\left(\mathbb{P}^{m}, d\right)$ and $\bar{Q}_{0,1}\left(\mathbb{P}^{m}, d\right)$ are empty by the definition of a stable quotient.

${ }^{9}$ The associated weights on $H^{0}\left(\mathbb{P}^{2}, \mathcal{O}_{\mathbb{P}^{2}}(1)\right)$ are $\lambda_{0}, \lambda_{1}, \lambda_{2}$ and so match the conventions of Section 0.3 .
} 
3.2. First correlators. We require several correlators defined via the Euler class of the obstruction bundle,

$$
e(\mathrm{Obs})=e\left(R^{1} \pi_{*} \mathrm{~S}^{3}\right)
$$

associated to the $K \mathbb{P}^{2}$ geometry on the moduli space $\bar{Q}_{g, n}\left(\mathbb{P}^{2}, d\right)$. The first two are obtained from standard stable quotient invariants. For $\gamma_{i} \in H_{\mathrm{T}}^{*}\left(\mathbb{P}^{2}\right)$, let

$$
\begin{aligned}
\left\langle\gamma_{1} \psi^{a_{1}}, \ldots, \gamma_{n} \psi^{a_{n}}\right\rangle_{g, n, d}^{\mathrm{SQ}} & =\int_{\left.\bar{Q}_{g, n}\left(\mathbb{P}^{2}, d\right)\right]^{\mathrm{vir}}} e(\mathrm{Obs}) \cdot \prod_{i=1}^{n} \mathrm{ev}_{i}^{*}\left(\gamma_{i}\right) \psi_{i}^{a_{i}}, \\
\left\langle\left\langle\gamma_{1} \psi^{a_{1}}, \ldots, \gamma_{n} \psi^{a_{n}}\right\rangle\right\rangle_{0, n}^{\mathrm{SQ}} & =\sum_{d \geq 0} \sum_{k \geq 0} \frac{q^{d}}{k !}\left\langle\gamma_{1} \psi^{a_{1}}, \ldots, \gamma_{n} \psi^{a_{n}}, t, \ldots, t\right\rangle_{0, n+k, d}^{\mathrm{SQ}}
\end{aligned}
$$

where, in the second series, $t \in H_{\mathrm{T}}^{*}\left(\mathbb{P}^{2}\right)$. We will systematically use the quasimap notation $0+$ for stable quotients,

$$
\begin{aligned}
\left\langle\gamma_{1} \psi^{a_{1}}, \ldots, \gamma_{n} \psi^{a_{n}}\right\rangle_{g, n, d}^{0+} & =\left\langle\gamma_{1} \psi^{a_{1}}, \ldots, \gamma_{n} \psi^{a_{n}}\right\rangle_{g, n, d}^{\mathrm{SQ}} \\
\left\langle\left\langle\gamma_{1} \psi^{a_{1}}, \ldots, \gamma_{n} \psi^{a_{n}}\right\rangle\right\rangle_{0, n}^{0+} & =\left\langle\left\langle\gamma_{1} \psi^{a_{1}}, \ldots, \gamma_{n} \psi^{a_{n}}\right\rangle\right\rangle_{0, n}^{\mathrm{SQ}} .
\end{aligned}
$$

3.3. Light markings. Moduli of quasimaps can be considered with $n$ ordinary (weight 1 ) markings and $k$ light (weight $\epsilon$ ) marking $\AA^{10}$,

$$
\bar{Q}_{g, n \mid k}^{0+, 0+}\left(\mathbb{P}^{2}, d\right) .
$$

Let $\gamma_{i} \in H_{\mathrm{T}}^{*}\left(\mathbb{P}^{2}\right)$ be equivariant cohomology classes, and let

$$
\delta_{j} \in H_{\mathrm{\top}}^{*}\left(\left[\mathbb{C}^{3} / \mathbb{C}^{*}\right]\right)
$$

be classes on the stack quotient. Following the notation of [23], we define series for the $K \mathbb{P}^{2}$ geometry,

$$
\begin{gathered}
\left\langle\gamma_{1} \psi^{a_{1}}, \ldots, \gamma_{n} \psi^{a_{n}} ; \delta_{1}, \ldots, \delta_{k}\right\rangle_{g, n \mid k, d}^{0+, 0+}= \\
\int_{\left.\left[\bar{Q}_{g, n \mid k}^{0+, 0+}\left(\mathbb{P}^{2}, d\right)\right]\right]^{\mathrm{vir}}} e(\mathrm{Obs}) \cdot \prod_{i=1}^{n} \mathrm{ev}_{i}^{*}\left(\gamma_{i}\right) \psi_{i}^{a_{i}} \cdot \prod_{j=1}^{k} \widehat{\mathrm{ev}}_{j}^{*}\left(\delta_{j}\right), \\
\left\langle\left\langle\gamma_{1} \psi^{a_{1}}, \ldots, \gamma_{n} \psi^{a_{n}}\right\rangle\right\rangle_{0, n}^{0+, 0+}= \\
\sum_{d \geq 0} \sum_{k \geq 0} \frac{q^{d}}{k !}\left\langle\gamma_{1} \psi^{a_{1}}, \ldots, \gamma_{n} \psi^{a_{n}} ; t, \ldots, t\right\rangle_{0, n \mid k, d}^{0+, 0+},
\end{gathered}
$$

\footnotetext{
${ }^{10}$ See Sections 2 and 5 of $[8$ ].
} 
where, in the second series, $t \in H_{\mathrm{T}}^{*}\left(\left[\mathbb{C}^{3} / \mathbb{C}^{*}\right]\right)$.

For each $\mathrm{T}$-fixed point $p_{i} \in \mathbb{P}^{2}$, let

$$
e_{i}=e\left(T_{p_{i}}\left(\mathbb{P}^{2}\right)\right) \cdot\left(-3 \lambda_{i}\right)
$$

be the equivariant Euler class of the tangent space of $K \mathbb{P}^{2}$ at $p_{i}$. Let

$$
\phi_{i}=\frac{-3 \lambda_{i} \prod_{j \neq i}\left(H-\lambda_{j}\right)}{e_{i}}, \quad \phi^{i}=e_{i} \phi_{i} \in H_{\mathrm{T}}^{*}\left(\mathbb{P}^{2}\right)
$$

be cycle classes. Crucial for us are the series

$$
\begin{aligned}
\mathbb{S}_{i}(\gamma) & =e_{i}\left\langle\left\langle\frac{\phi_{i}}{z-\psi}, \gamma\right\rangle\right\rangle_{0,2}^{0+, 0+} \\
\mathbb{V}_{i j} & =\left\langle\left\langle\frac{\phi_{i}}{x-\psi}, \frac{\phi_{j}}{y-\psi}\right\rangle\right\rangle_{0,2}^{0+, 0+} .
\end{aligned}
$$

Unstable degree 0 terms are included by hand in the above formulas. For $\mathbb{S}_{i}(\gamma)$, the unstable degree 0 term is $\left.\gamma\right|_{p_{i}}$. For $\mathbb{V}_{i j}$, the unstable degree 0 term is $\frac{\delta_{i j}}{e_{i}(x+y)}$.

We also write

$$
\mathbb{S}(\gamma)=\sum_{i=0}^{2} \phi_{i} \mathbb{S}_{i}(\gamma)
$$

The series $\mathbb{S}_{i}$ and $\mathbb{V}_{i j}$ satisfy the basic relation

$$
e_{i} \mathbb{V}_{i j}(x, y) e_{j}=\frac{\left.\left.\sum_{k=0}^{2} \mathbb{S}_{i}\left(\phi_{k}\right)\right|_{z=x} \mathbb{S}_{j}\left(\phi^{k}\right)\right|_{z=y}}{x+y}
$$

proven ${ }^{11}$ in 10 .

Associated to each T-fixed point $p_{i} \in \mathbb{P}^{2}$, there is a special T-fixed point locus,

$$
\bar{Q}_{0, k \mid m}^{0+, 0+}\left(\mathbb{P}^{2}, d\right)^{\top, p_{i}} \subset \bar{Q}_{0, k \mid m}^{0+, 0+}\left(\mathbb{P}^{2}, d\right),
$$

where all markings lie on a single connected genus 0 domain component contracted to $p_{i}$. Let Nor denote the equivariant normal bundle of $Q_{0, n \mid k}^{0+, 0+}\left(\mathbb{P}^{2}, d\right)^{\top}, p_{i}$ with respect to the embedding (21). Define

$$
\begin{gathered}
\left\langle\gamma_{1} \psi^{a_{1}}, \ldots, \gamma_{n} \psi^{a_{n}} ; \delta_{1}, \ldots, \delta_{k}\right\rangle_{0, n \mid k, d}^{0+, 0+, p_{i}}= \\
\int_{\left[\bar{Q}_{0, n \mid k}^{0+, 0+}\left(\mathbb{P}^{2}, d\right)^{\left.\mathrm{T}, p_{i}\right]}\right.} \frac{e(\mathrm{Obs})}{e(\mathrm{Nor})} \cdot \prod_{i=1}^{n} \operatorname{ev}_{i}^{*}\left(\gamma_{i}\right) \psi_{i}^{a_{i}} \cdot \prod_{j=1}^{k} \widehat{\operatorname{ev}}_{j}^{*}\left(\delta_{j}\right),
\end{gathered}
$$

\footnotetext{
${ }^{11}$ In Gromov-Witten theory, a parallel relation is obtained immediately from the WDDV equation and the string equation. Since the map forgetting a point is not always well-defined for quasimaps, a different argument is needed here [10.
} 


$$
\begin{aligned}
\left\langle\left\langle\gamma_{1} \psi^{a_{1}}, \ldots, \gamma_{n} \psi^{a_{n}}\right\rangle\right\rangle_{0, n}^{0+, 0+, p_{i}} & = \\
& \sum_{d \geq 0} \sum_{k \geq 0} \frac{q^{d}}{k !}\left\langle\gamma_{1} \psi^{a_{1}}, \ldots, \gamma_{n} \psi^{a_{n}} ; t, \ldots, t\right\rangle_{0, n \mid k, \beta}^{0+, 0+, p_{i}} .
\end{aligned}
$$

\subsection{Graph spaces and I-functions.}

3.4.1. Graph spaces. The big I-function is defined in [8] via the geometry of weighted quasimap graph spaces. We briefly summarize the constructions of [8] in the special case of $(0+, 0+)$-stability. The more general weightings discussed in [8] will not be needed here.

As in Section 3.3, we consider the quotient

$$
\mathbb{C}^{3} / \mathbb{C}^{*}
$$

associated to $\mathbb{P}^{2}$. Following [8], there is a $(0+, 0+)$-stable quasimap graph space

$$
\mathrm{QG}_{g, n \mid k, d}^{0+, 0+}\left(\left[\mathbb{C}^{3} / \mathbb{C}^{*}\right]\right) \text {. }
$$

A $\mathbb{C}$-point of the graph space is described by data

$$
\left((C, \mathbf{x}, \mathbf{y}),(f, \varphi): C \longrightarrow\left[\mathbb{C}^{3} / \mathbb{C}^{*}\right] \times\left[\mathbb{C}^{2} / \mathbb{C}^{*}\right]\right) .
$$

By the definition of stability, $\varphi$ is a regular map to

$$
\mathbb{P}^{1}=\mathbb{C}^{2} / / \mathbb{C}^{*}
$$

of class 1 . Hence, the domain curve $C$ has a distinguished irreducible component $C_{0}$ canonically isomorphic to $\mathbb{P}^{1}$ via $\varphi$. The standard $\mathbb{C}^{*}$ action,

$$
t \cdot\left[\xi_{0}, \xi_{1}\right]=\left[t \xi_{0}, \xi_{1}\right], \quad \text { for } t \in \mathbb{C}^{*},\left[\xi_{0}, \xi_{1}\right] \in \mathbb{P}^{1},
$$

induces a $\mathbb{C}^{*}$-action on the graph space.

The $\mathbb{C}^{*}$-equivariant cohomology of a point is a free algebra with generator $z$,

$$
H_{\mathbb{C}^{*}}^{*}(\operatorname{Spec}(\mathbb{C}))=\mathbb{Q}[z] .
$$

Our convention is to define $z$ as the $\mathbb{C}^{*}$-equivariant first Chern class of the tangent line $T_{0} \mathbb{P}^{1}$ at $0 \in \mathbb{P}^{1}$ with respect to the action (23),

$$
z=c_{1}\left(T_{0} \mathbb{P}^{1}\right) \text {. }
$$

The $\mathrm{T}$-action on $\mathbb{C}^{3}$ lifts to a $\mathrm{T}$-action on the graph space 22 which commutes with the $\mathbb{C}^{*}$-action obtained from the distinguished domain 
component. As a result, we have a $T \times \mathbb{C}^{*}$-action on the graph space and $\mathrm{T} \times \mathbb{C}^{*}$-equivariant evaluation morphisms

$$
\begin{array}{ll}
\mathrm{ev}_{i}: \mathrm{QG}_{g, n \mid k, \beta}^{0+, 0+}\left(\left[\mathbb{C}^{3} / \mathbb{C}^{*}\right]\right) \rightarrow \mathbb{P}^{2}, & i=1, \ldots, n, \\
\widehat{\mathrm{ev}}_{j}: \mathrm{QG}_{g, n \mid k, \beta}^{0+, 0+}\left(\left[\mathbb{C}^{3} / \mathbb{C}^{*}\right]\right) \rightarrow\left[\mathbb{C}^{3} / \mathbb{C}^{*}\right], & j=1, \ldots, k .
\end{array}
$$

Since a morphism

$$
f: C \rightarrow\left[\mathbb{C}^{3} / \mathbb{C}^{*}\right]
$$

is equivalent to the data of a principal G-bundle $P$ on $C$ and a section $u$ of $P \times_{\mathbb{C}^{*}} \mathbb{C}^{3}$, there is a natural morphism

$$
C \rightarrow E \mathbb{C}^{*} \times \mathbb{C}^{*} \mathbb{C}^{3}
$$

and hence a pull-back map

$$
f^{*}: H_{\mathbb{C}^{*}}^{*}\left(\left[\mathbb{C}^{3} / \mathbb{C}^{*}\right]\right) \rightarrow H^{*}(C) .
$$

The above construction applied to the universal curve over the moduli space and the universal morphism to $\left[\mathbb{C}^{3} / \mathbb{C}^{*}\right]$ is T-equivariant. Hence, we obtain a pull-back map

$$
\widehat{\mathrm{ev}}_{j}^{*}: H_{\mathrm{T}}^{*}\left(\mathbb{C}^{3}, \mathbb{Q}\right) \otimes_{\mathbb{Q}} \mathbb{Q}[z] \rightarrow H_{\mathrm{T} \times \mathbb{C}^{*}}^{*}\left(\mathrm{QG}_{g, n \mid k, \beta}^{0+, 0+}\left(\left[\mathbb{C}^{3} / \mathbb{C}^{*}\right]\right), \mathbb{Q}\right)
$$

associated to the evaluation map $\widehat{\mathrm{ev}}_{j}$.

3.4.2. I-functions. The description of the fixed loci for the $\mathbb{C}^{*}$-action on

$$
\mathrm{QG}_{g, 0 \mid k, d}^{0+, 0+}\left(\left[\mathbb{C}^{3} / \mathbb{C}^{*}\right]\right)
$$

is parallel to the description in [7, §4.1] for the unweighted case. In particular, there is a distinguished subset $\mathbf{M}_{k, d}$ of the $\mathbb{C}^{*}$-fixed locus for which all the markings and the entire curve class $d$ lie over $0 \in \mathbb{P}^{1}$. The locus $\mathrm{M}_{k, d}$ comes with a natural proper evaluation map $e v_{\bullet}$ obtained from the generic point of $\mathbb{P}^{1}$ :

$$
\mathrm{ev}_{\bullet}: \mathrm{M}_{k, d} \rightarrow \mathbb{C}^{3} / / \mathbb{C}^{*}=\mathbb{P}^{2}
$$

We can explicitly write

$$
\mathrm{M}_{k, d} \cong \mathrm{M}_{d} \times 0^{k} \subset \mathrm{M}_{d} \times\left(\mathbb{P}^{1}\right)^{k},
$$

where $\mathrm{M}_{d}$ is the $\mathbb{C}^{*}$-fixed locus in $\mathrm{QG}_{0,0, d}^{0+}\left(\left[\mathbb{C}^{3} / \mathbb{C}^{*}\right]\right)$ for which the class $d$ is concentrated over $0 \in \mathbb{P}^{1}$. The locus $\mathrm{M}_{d}$ parameterizes quasimaps of class $d$,

$$
f: \mathbb{P}^{1} \longrightarrow\left[\mathbb{C}^{3} / \mathbb{C}^{*}\right]
$$

with a base-point of length $d$ at $0 \in \mathbb{P}^{1}$. The restriction of $f$ to $\mathbb{P}^{1} \backslash\{0\}$ is a constant map to $\mathbb{P}^{2}$ defining the evaluation map ev. 
As in [6, 7, 11, we define the big I-function as the generating function for the push-forward via $e v$. of localization residue contributions of $\mathrm{M}_{k, d}$. For $\mathbf{t} \in H_{\mathrm{T}}^{*}\left(\left[\mathbb{C}^{3} / \mathbb{C}^{*}\right], \mathbb{Q}\right) \otimes_{\mathbb{Q}} \mathbb{Q}[z]$, let

$$
\begin{aligned}
\operatorname{Res}_{\mathrm{M}_{k, d}}\left(\mathbf{t}^{k}\right) & =\prod_{j=1}^{k} \widehat{\operatorname{ev}}_{j}^{*}(\mathbf{t}) \cap \operatorname{Res}_{\mathrm{M}_{k, d}}\left[\mathrm{QG}_{g, 0 \mid k, d}^{0+, 0+}\left(\left[\mathbb{C}^{3} / \mathbb{C}^{*}\right]\right)\right]^{\mathrm{vir}} \\
& =\frac{\prod_{j=1}^{k} \widehat{\mathrm{ev}}_{j}^{*}(\mathbf{t}) \cap\left[\mathrm{M}_{k, d}\right]}{\mathrm{e}\left(\operatorname{Nor}_{\mathrm{M}_{k, d}}^{\mathrm{vir}}\right)}
\end{aligned}
$$

where $\operatorname{Nor}_{\mathbf{M}_{k, d}}^{\mathrm{vir}}$ is the virtual normal bundle.

Definition 5. The big I-function for the $(0+, 0+)$-stability condition, as a formal function in $\mathbf{t}$, is

$$
\mathrm{I}(q, \mathbf{t}, z)=\sum_{d \geq 0} \sum_{k \geq 0} \frac{q^{d}}{k !} \mathrm{ev}_{\bullet *}\left(\operatorname{Res}_{\mathrm{M}_{k, d}}\left(\mathbf{t}^{k}\right)\right) .
$$

3.4.3. Evaluations. Let $\widetilde{H} \in H_{\mathrm{\top}}^{*}\left(\left[\mathbb{C}^{3} / \mathbb{C}^{*}\right]\right)$ and $H \in H_{\mathrm{\top}}^{*}\left(\mathbb{P}^{2}\right)$ denote the respective hyperplane classes. The I-function of Definition 5 is evaluated in [8].

Proposition 6. For $\mathbf{t}=t \widetilde{H} \in H_{\mathrm{T}}^{*}\left(\left[\mathbb{C}^{3} / \mathbb{C}^{*}\right], \mathbb{Q}\right)$,

$$
\mathbb{I}(t)=\sum_{d=0}^{\infty} q^{d} e^{t(H+d z) / z} \frac{\prod_{k=0}^{3 d-1}(-3 H-k z)}{\prod_{i=0}^{2} \prod_{k=1}^{d}\left(H-\lambda_{i}+k z\right)} .
$$

We return now to the functions $\mathbb{S}_{i}(\gamma)$ defined in Section 3.3. Using Birkhoff factorization, an evaluation of the series $\mathbb{S}\left(H^{j}\right)$ can be obtained from the I-function, see [23]:

$$
\begin{aligned}
\mathbb{S}(1) & =\mathbb{I}, \\
\mathbb{S}(H) & =\frac{z \frac{d}{d t} \mathbb{S}(1)}{\left.z \frac{d}{d t} \mathbb{S}(1)\right|_{t=0, H=1, z=\infty}}, \\
\mathbb{S}\left(H^{2}\right) & =\frac{z \frac{d}{d t} \mathbb{S}(H)}{\left.z \frac{d}{d t} \mathbb{S}(H)\right|_{t=0, H=1, z=\infty}} .
\end{aligned}
$$

For a series $F \in \mathbb{C}\left[\left[\frac{1}{z}\right]\right]$, the specialization $\left.F\right|_{z=\infty}$ denotes constant term of $F$ with respect to $\frac{1}{z}$.

3.4.4. Further calculations. Define small $I$-function

$$
\overline{\mathbb{I}}(q) \in H_{\mathrm{T}}^{*}\left(\mathbb{P}^{2}, \mathbb{Q}\right)[[q]]
$$


by the restriction

$$
\overline{\mathbb{I}}(q)=\left.\mathbb{I}(q, t)\right|_{t=0} .
$$

Define differential operators

$$
\mathrm{D}=q \frac{d}{d q}, \quad M=H+z \mathrm{D} .
$$

Applying $z \frac{d}{d t}$ to $\mathbb{I}$ and then restricting to $t=0$ has same effect as applying $M$ to $\overline{\mathbb{I}}$

$$
\left.\left[\left(z \frac{d}{d t}\right)^{k} \mathbb{I}\right]\right|_{t=0}=M^{k} \overline{\mathrm{I}}
$$

The function $\overline{\mathbb{I}}$ satisfies following Picard-Fuchs equation

$$
\left(M^{3}-\lambda_{0}^{3}+3 q M(3 M+z)(3 M+2 z)\right) \overline{\mathbb{I}}=0
$$

implied by the Picard-Fuchs equation for II,

$$
\left(\left(z \frac{d}{d t}\right)^{3}-\lambda_{0}^{3}+3 q\left(z \frac{d}{d t}\right)\left(3\left(z \frac{d}{d t}\right)+z\right)\left(3\left(z \frac{d}{d t}\right)+2 z\right)\right) \mathbb{I}=0 .
$$

The restriction $\left.\overline{\mathbb{I}}\right|_{H=\lambda_{i}}$ admits following asymptotic form

$$
\left.\overline{\mathbb{I}}\right|_{H=\lambda_{i}}=e^{\mu \lambda_{i} / z}\left(R_{0}+R_{1}\left(\frac{z}{\lambda_{i}}\right)+R_{2}\left(\frac{z}{\lambda_{i}}\right)^{2}+\ldots\right)
$$

with series $\mu, R_{k} \in \mathbb{C}[[q]]$.

A derivation of (27) is obtained in [33] via the Picard-Fuchs equation (26) for $\left.\overline{\mathrm{I}}\right|_{H=\lambda_{i}}$. The series $\mu$ and $R_{k}$ are found by solving differential equations obtained from the coefficient of $z^{k}$. For example,

$$
\begin{aligned}
1+\mathrm{D} \mu & =L, \\
R_{0} & =1, \\
R_{1} & =\frac{1}{18}\left(1-L^{2}\right), \\
R_{2} & =\frac{1}{648}\left(1-24 L-2 L^{2}+25 L^{4}\right),
\end{aligned}
$$

where $L(q)=(1+27 q)^{-1 / 3}$. The specialization (19) is used for these results.

In [33, the authors study the $I$-function ${ }^{12}$ related to Calabi-Yau hypersurfaces in $\mathbb{P}^{m}$. The local geometry $K \mathbb{P}^{2}$ here has a slightly different $I$-function from the function $\mathcal{F}$ in [33] for the cubic in $\mathbb{P}^{2}$. More

\footnotetext{
${ }^{12}$ The $I$-function is $\mathcal{F}$ in the notation of [33].
} 
precisely,

$$
\mathcal{F}_{-1}(q)=\overline{\mathbb{I}}(-q)
$$

We can either apply the methods of 33 to our $I$-functions, or we can just take account of the difference between the two $I$-functions (which amounts to a shifting of indices). The latter is easier.

Define the series $C_{1}$ and $C_{2}$ by the equations

$$
\begin{aligned}
& C_{1}=\left.z \frac{d}{d t} \mathbb{S}(1)\right|_{z=\infty, t=0, H=1} \\
& C_{2}=\left.z \frac{d}{d t} \mathbb{S}(H)\right|_{z=\infty, t=0, H=1} . \\
& C_{0}=\left.z \frac{d}{d t} \mathbb{S}\left(H^{2}\right)\right|_{z=\infty, t=0, H=1} .
\end{aligned}
$$

The above series are equal (up to sign) to $I_{0}, I_{1}$ and $I_{2}$ respectively in the notation of [33] for the cubic in $\mathbb{P}^{2}$. The following relations were proven in [33],

$$
\begin{aligned}
C_{0} C_{1} C_{2} & =(1+27 q)^{-1}, \\
C_{0} & =C_{1} .
\end{aligned}
$$

From the equations 25) and (27), we can show the series

$$
\overline{\mathbb{S}}_{i}(1)=\left.\overline{\mathbb{S}}(1)\right|_{H=\lambda_{i}}, \quad \overline{\mathbb{S}}_{i}(H)=\left.\overline{\mathbb{S}}(H)\right|_{H=\lambda_{i}}, \quad \overline{\mathbb{S}}_{i}\left(H^{2}\right)=\left.\overline{\mathbb{S}}\left(H^{2}\right)\right|_{H=\lambda_{i}}
$$

have the following asymptotic expansions:

$$
\begin{aligned}
\overline{\mathrm{S}}_{i}(1) & =e^{\frac{\mu \lambda_{i}}{z}}\left(R_{00}+R_{01}\left(\frac{z}{\lambda_{i}}\right)+R_{02}\left(\frac{z}{\lambda_{i}}\right)^{2}+\ldots\right), \\
\overline{\mathrm{S}}_{i}(H) & =e^{\frac{\mu \lambda_{i}}{z}} \frac{L \lambda_{i}}{C_{1}}\left(R_{10}+R_{11}\left(\frac{z}{\lambda_{i}}\right)+R_{12}\left(\frac{z}{\lambda_{i}}\right)^{2}+\ldots\right), \\
\overline{\mathbb{S}}_{i}\left(H^{2}\right) & =e^{\frac{\mu \lambda_{i}}{z}} \frac{L^{2} \lambda_{i}^{2}}{C_{1} C_{2}}\left(R_{20}+R_{21}\left(\frac{z}{\lambda_{i}}\right)+R_{22}\left(\frac{z}{\lambda_{i}}\right)^{2}+\ldots\right) .
\end{aligned}
$$

We follow here the normalization of [33]. Note

$$
R_{0 k}=R_{k} .
$$

As in [33, Theorem 4], we obtain the following constraints.

Proposition 7. (Zagier-Zinger [33]) For all $k \geq 0$, we have

$$
R_{k} \in \mathbb{C}\left[L^{ \pm 1}\right]
$$

Similarly, we also obtain results for $\left.\overline{\mathbb{S}}(H)\right|_{H=\lambda_{i}}$ and $\left.\overline{\mathbb{S}}\left(H^{2}\right)\right|_{H=\lambda_{i}}$. 
Lemma 8. For all $k \geq 0$, we have

$$
\begin{aligned}
& R_{1 k} \in \mathbb{C}\left[L^{ \pm 1}\right] \\
& R_{2 k}=Q_{2 k}-\frac{R_{1 k-1}}{L} X
\end{aligned}
$$

with $Q_{2 k} \in \mathbb{C}\left[L^{ \pm 1}\right]$ and $X=\frac{\mathrm{D} C_{1}}{C_{1}}$.

3.5. Determining $D X$. Using Birkhoff factorization of 25 further, we have

$$
\mathbb{S}\left(H^{3}\right)=\frac{z \frac{d}{d t} \mathbb{S}\left(H^{2}\right)}{\left.z \frac{d}{d t} \mathbb{S}\left(H^{2}\right)\right|_{t=0, H=1, z=\infty}} .
$$

Since $H^{3}=\lambda_{0}^{3}$ with the specialization 190 , we have

$$
\mathbb{S}(1)=\frac{1}{\lambda_{0}^{3}} \mathbb{S}\left(H^{3}\right) \text {. }
$$

From (25) and (31), we obtain the following result.

Lemma 9. We have

$$
\begin{aligned}
& R_{1 p+1}=R_{0 p+1}+\frac{\mathrm{D} R_{0 p}}{L} \\
& R_{2 p+1}=R_{1 p+1}+\frac{\mathrm{D} R_{1 p}}{L}+\left(\frac{\mathrm{D} L}{L^{2}}-\frac{X}{L}\right) R_{1 p} \\
& R_{0 p+1}=R_{2 p+1}+\frac{\mathrm{D} R_{2 p}}{L}-\left(\frac{\mathrm{D} L}{L^{2}}-\frac{X}{L}\right) R_{2 p} .
\end{aligned}
$$

After setting $p=1$ in Lemma 9, we find

$$
X^{2}-\left(L^{3}-1\right) X+\mathrm{D} X-\frac{2}{9}\left(L^{3}-1\right)=0 .
$$

By the above result, the differential ring

$$
\mathbb{C}\left[L^{ \pm 1}\right][X, \mathrm{D} X, \mathrm{DD} X, \ldots]
$$

is just the polynomial ring $\mathbb{C}\left[L^{ \pm 1}\right][X]$.

We can find the value of $R_{2 k}$ in terms of $R_{k}$ using Lemma 9 .

$$
R_{2 p+2}=R_{p+2}+2 \frac{\mathrm{D} R_{p+1}}{L}+\frac{\mathrm{D} L}{L^{2}} R_{p+1}+\frac{\mathrm{D}^{2} R_{p}}{L^{2}}-\left(\frac{R_{p+1}}{L}+\frac{\mathrm{D} R_{p}}{L^{2}}\right) X
$$




\section{Higher GeNUS SERIES ON $\bar{M}_{g, n}$}

4.1. Intersection theory on $\bar{M}_{g, n}$. We review here the now standard method used by Givental [20, 21, 26] to express genus $g$ descendent correlators in terms of genus 0 data.

Let $t_{0}, t_{1}, t_{2}, \ldots$ be formal variables. The series

$$
T(c)=t_{0}+t_{1} c+t_{2} c^{2}+\ldots
$$

in the additional variable $c$ plays a basic role. The variable $c$ will later be replaced by the first Chern class $\psi_{i}$ of a cotangent line over $\bar{M}_{g, n}$,

$$
T\left(\psi_{i}\right)=t_{0}+t_{1} \psi_{i}+t_{2} \psi_{i}^{2}+\ldots,
$$

with the index $i$ depending on the position of the series $T$ in the correlator.

Let $2 g-2+n>0$. For $a_{i} \in \mathbb{Z}_{\geq 0}$ and $\gamma \in H^{*}\left(\bar{M}_{g, n}\right)$, define the correlator

$$
\left\langle\left\langle\psi^{a_{1}}, \ldots, \psi^{a_{n}} \mid \gamma\right\rangle\right\rangle_{g, n}=\sum_{k \geq 0} \frac{1}{k !} \int_{\bar{M}_{g, n+k}} \gamma \psi_{1}^{a_{1}} \cdots \psi_{n}^{a_{n}} \prod_{i=1}^{k} T\left(\psi_{n+i}\right) .
$$

In the above summation, the $k=0$ term is

$$
\int_{\bar{M}_{g, n}} \gamma \psi_{1}^{a_{1}} \cdots \psi_{n}^{a_{n}}
$$

We also need the following correlator defined for the unstable case,

$$
\langle\langle 1,1\rangle\rangle_{0,2}=\sum_{k>0} \frac{1}{k !} \int_{\bar{M}_{0,2+k}} \prod_{i=1}^{k} T\left(\psi_{2+i}\right) .
$$

For formal variables $x_{1}, \ldots, x_{n}$, we also define the correlator

$$
\left\langle\left\langle\frac{1}{x_{1}-\psi}, \ldots, \frac{1}{x_{n}-\psi} \mid \gamma\right\rangle\right\rangle_{g, n}
$$

in the standard way by expanding $\frac{1}{x_{i}-\psi}$ as a geometric series.

Denote by $\mathbb{L}$ the differential operator

$$
\mathbb{L}=\frac{\partial}{\partial t_{0}}-\sum_{i=1}^{\infty} t_{i} \frac{\partial}{\partial t_{i-1}}=\frac{\partial}{\partial t_{0}}-t_{1} \frac{\partial}{\partial t_{0}}-t_{2} \frac{\partial}{\partial t_{1}}-\ldots
$$

The string equation yields the following result. 
Lemma 10. For $2 g-2+n>0$, we have $\mathbb{L}\langle\langle 1, \ldots, 1 \mid \gamma\rangle\rangle_{g, n}=0$ and

$$
\begin{aligned}
\mathbb{L}\left\langle\left\langle\frac{1}{x_{1}-\psi}, \ldots, \frac{1}{x_{n}-\psi} \mid \gamma\right\rangle\right\rangle_{g, n} & = \\
& \left(\frac{1}{x_{1}}+\ldots+\frac{1}{x_{n}}\right)\left\langle\left\langle\frac{1}{x_{1}-\psi}, \ldots \frac{1}{x_{n}-\psi} \mid \gamma\right\rangle\right\rangle_{g, n} .
\end{aligned}
$$

After the restriction $t_{0}=0$ and application of the dilaton equation, the correlators are expressed in terms of finitely many integrals (by the dimension constraint). For example,

$$
\begin{aligned}
\left.\langle\langle 1,1,1\rangle\rangle_{0,3}\right|_{t_{0}=0} & =\frac{1}{1-t_{1}}, \\
\left.\langle\langle 1,1,1,1\rangle\rangle_{0,4}\right|_{t_{0}=0} & =\frac{t_{2}}{\left(1-t_{1}\right)^{3}}, \\
\left.\langle\langle 1,1,1,1,1\rangle\rangle_{0,5}\right|_{t_{0}=0} & =\frac{t_{3}}{\left(1-t_{1}\right)^{4}}+\frac{3 t_{2}^{2}}{\left(1-t_{1}\right)^{5}}, \\
\left.\langle\langle 1,1,1,1,1,1\rangle\rangle_{0,6}\right|_{t_{0}=0} & =\frac{t_{4}}{\left(1-t_{1}\right)^{5}}+\frac{10 t_{2} t_{3}}{\left(1-t_{1}\right)^{6}}+\frac{15 t_{2}^{3}}{\left(1-t_{1}\right)^{7}} .
\end{aligned}
$$

We consider $\mathbb{C}\left(t_{1}\right)\left[t_{2}, t_{3}, \ldots\right]$ as $\mathbb{Z}$-graded ring over $\mathbb{C}\left(t_{1}\right)$ with

$$
\operatorname{deg}\left(t_{i}\right)=i-1 \text { for } i \geq 2 \text {. }
$$

Define a subspace of homogeneous elements by

$$
\mathbb{C}\left[\frac{1}{1-t_{1}}\right]\left[t_{2}, t_{3}, \ldots\right]_{\text {Hom }} \subset \mathbb{C}\left(t_{1}\right)\left[t_{2}, t_{3}, \ldots\right] .
$$

We easily see

$$
\left.\left\langle\left\langle\psi^{a_{1}}, \ldots, \psi^{a_{n}} \mid \gamma\right\rangle\right\rangle_{g, n}\right|_{t_{0}=0} \in \mathbb{C}\left[\frac{1}{1-t_{1}}\right]\left[t_{2}, t_{3}, \ldots\right]_{\mathrm{Hom}} .
$$

Using the leading terms (of lowest degree in $\frac{1}{\left(1-t_{1}\right)}$ ), we obtain the following result.

Lemma 11. The set of genus 0 correlators

$$
\left\{\left.\langle\langle 1, \ldots, 1\rangle\rangle_{0, n}\right|_{t_{0}=0}\right\}_{n \geq 4}
$$

freely generate the ring $\mathbb{C}\left(t_{1}\right)\left[t_{2}, t_{3}, \ldots\right]$ over $\mathbb{C}\left(t_{1}\right)$.

By Lemma 11, we can find a unique representation of $\left.\left\langle\left\langle\psi^{a_{1}}, \ldots, \psi^{a_{n}}\right\rangle\right\rangle_{g, n}\right|_{t_{0}=0}$ in the variables

$$
\left\{\left.\langle\langle 1, \ldots, 1\rangle\rangle_{0, n}\right|_{t_{0}=0}\right\}_{n \geq 3}
$$


The $n=3$ correlator is included in the set 35 to capture the variable $t_{1}$. For example, in $g=1$,

$$
\begin{aligned}
\left.\langle\langle 1,1\rangle\rangle_{1,2}\right|_{t_{0}=0} & =\frac{1}{24}\left(\frac{\left.\langle\langle 1,1,1,1,1\rangle\rangle_{0,5}\right|_{t_{0}=0}}{\langle 1,1,1\rangle\rangle\left._{0,3}\right|_{t_{0}=0}}-\frac{\left.\langle\langle 1,1,1,1\rangle\rangle_{0,4}^{2}\right|_{t_{0}=0}}{\left.\langle\langle 1,1,1\rangle\rangle_{0,3}^{2}\right|_{t_{0}=0}}\right), \\
\left.\langle\langle 1\rangle\rangle_{1,1}\right|_{t_{0}=0} & =\frac{1}{24} \frac{\left.\langle\langle 1,1,1,1\rangle\rangle_{0,4}\right|_{t_{0}=0}}{\left.\langle\langle 1,1,1\rangle\rangle_{0,3}\right|_{t_{0}=0}}
\end{aligned}
$$

A more complicated example in $g=2$ is

$$
\begin{aligned}
\left.\langle\langle\rangle\rangle_{2,0}\right|_{t_{0}=0}= & \frac{1}{1152} \frac{\left.\langle\langle 1,1,1,1,1,1\rangle\rangle_{0,6}\right|_{t_{0}=0}}{\left.\langle\langle 1,1,1\rangle\rangle_{0,3}\right|_{t_{0}=0} ^{2}} \\
& -\frac{7}{1920} \frac{\left.\left.\langle\langle 1,1,1,1,1\rangle\rangle_{0,5}\right|_{t_{0}=0}\langle\langle 1,1,1,1\rangle\rangle_{0,4}\right|_{t_{0}=0}}{\left.\langle\langle 1,1,1\rangle\rangle_{0,3}\right|_{t_{0}=0} ^{3}} \\
& +\frac{1}{360} \frac{\left.\langle\langle 1,1,1,1\rangle\rangle_{0,4}\right|_{t_{0}=0} ^{3}}{\left.\langle\langle 1,1,1\rangle\rangle_{0,3}\right|_{t_{0}=0} ^{4}} .
\end{aligned}
$$

Definition 12. For $\gamma \in H^{*}\left(\bar{M}_{g, k}\right)$, let

$$
\mathrm{P}_{g, n}^{a_{1}, \ldots, a_{n}, \gamma}\left(s_{0}, s_{1}, s_{2}, \ldots\right) \in \mathbb{Q}\left(s_{0}, s_{1}, . .\right)
$$

be the unique rational function satisfying the condition

$$
\left.\left\langle\left\langle\psi^{a_{1}}, \ldots, \psi^{a_{n}} \mid \gamma\right\rangle\right\rangle_{g, n}\right|_{t_{0}=0}=\left.\mathrm{P}_{g, n}^{a_{1}, a_{2}, \ldots, a_{n}, \gamma}\right|_{s_{i}=\left.\langle\langle 1, \ldots, 1\rangle\rangle_{0, i+3}\right|_{t_{0}=0}} .
$$

Proposition 13. For $2 g-2+n>0$, we have

$$
\langle\langle 1, \ldots, 1 \mid \gamma\rangle\rangle_{g, n}=\left.\mathrm{P}_{g, n}^{0, \ldots, 0, \gamma}\right|_{s_{i}=\langle\langle 1, \ldots, 1\rangle\rangle_{0, i+3}} .
$$

Proof. Both sides of the equation satisfy the differential equation

$$
\mathbb{L}=0 \text {. }
$$

By definition, both sides have the same initial conditions at $t_{0}=0$.

Proposition 14. For $2 g-2+n>0$,

$$
\begin{aligned}
&\left\langle\left\langle\frac{1}{x_{1}-\psi_{1}}, \ldots, \frac{1}{x_{n}-\psi_{n}} \mid \gamma\right\rangle\right\rangle_{g, n}= \\
& e^{\langle\langle 1,1\rangle\rangle_{0,2}\left(\sum_{i} \frac{1}{x_{i}}\right)} \sum_{a_{1}, \ldots, a_{n}} \frac{\left.P_{g, n}^{a_{1}, \ldots, a_{n}, \gamma}\right|_{s_{i}=\langle\langle 1, \ldots, 1\rangle\rangle_{0, i+3}}}{x_{1}^{a_{1}+1} \cdots x_{n}^{a_{n}+1}} .
\end{aligned}
$$

Proof. Both sides of the equation satisfy differential equation

$$
\mathrm{L}-\sum_{i} \frac{1}{x_{i}}=0 .
$$

Both sides have the same initial conditions at $t_{0}=0$. We use here

$$
\mathbb{L}\langle\langle 1,1\rangle\rangle_{0,2}=1,\left.\quad\langle\langle 1,1\rangle\rangle_{0,2}\right|_{t_{0}=0}=0 .
$$


There is no conflict here with Lemma 10 since $(g, n)=(0,2)$ is not in the stable range.

4.2. The unstable case $(0,2)$. The definition given in (34) of the correlator is valid in the stable range

$$
2 g-2+n>0 \text {. }
$$

The unstable case $(g, n)=(0,2)$ plays a special role. We define

$$
\left\langle\left\langle\frac{1}{x_{1}-\psi_{1}}, \frac{1}{x_{2}-\psi_{2}}\right\rangle\right\rangle_{0,2}
$$

by adding the degenerate term

$$
\frac{1}{x_{1}+x_{2}}
$$

to the terms obtained by the expansion of $\frac{1}{x_{i}-\psi_{i}}$ as a geometric series. The degenerate term is associated to the (unstable) moduli space of genus 0 with 2 markings.

Proposition 15. We have

$$
\left\langle\left\langle\frac{1}{x_{1}-\psi_{1}}, \frac{1}{x_{2}-\psi_{2}}\right\rangle\right\rangle_{0,2}=e^{\langle\langle 1,1\rangle\rangle_{0,2}\left(\frac{1}{x_{1}}+\frac{1}{x_{2}}\right)}\left(\frac{1}{x_{1}+x_{2}}\right) .
$$

Proof. Both sides of the equation satisfy differential equation

$$
\mathbb{L}-\sum_{i=1}^{2} \frac{1}{x_{i}}=0
$$

Both sides have the same initial conditions at $t_{0}=0$.

4.3. Local invariants and wall crossing. The torus $\mathrm{T}$ acts on the moduli spaces $\bar{M}_{g, n}\left(\mathbb{P}^{2}, d\right)$ and $\bar{Q}_{g, n}\left(\mathbb{P}^{2}, d\right)$. We consider here special localization contributions associated to the fixed points $p_{i} \in \mathbb{P}^{2}$.

Consider first the moduli of stable maps. Let

$$
\bar{M}_{g, n}\left(\mathbb{P}^{2}, d\right)^{\top, p_{i}} \subset \bar{M}_{g, n}\left(\mathbb{P}^{2}, d\right)
$$

be the union of T-fixed loci which parameterize stable maps obtained by attaching T-fixed rational tails to a genus $g, n$-pointed DeligneMumford stable curve contracted to the point $p_{i} \in \mathbb{P}^{2}$. Similarly, let

$$
\bar{Q}_{g, n}\left(\mathbb{P}^{2}, d\right)^{\top, p_{i}} \subset \bar{Q}_{g, n}\left(\mathbb{P}^{2}, d\right)
$$

be the parallel T-fixed locus parameterizing stable quotients obtained by attaching base points to a genus $g, n$-pointed Deligne-Mumford stable curve contracted to the point $p_{i} \in \mathbb{P}^{2}$. 
Let $\Lambda_{i}$ denote the localization of the ring

$$
\mathbb{C}\left[\lambda_{0}^{ \pm 1}, \lambda_{1}^{ \pm 1}, \lambda_{2}^{ \pm 1}\right]
$$

at the three tangent weights at $p_{i} \in \mathbb{P}^{2}$. Using the virtual localization formula [22], there exist unique series

$$
S_{p_{i}} \in \Lambda_{i}[\psi][[Q]]
$$

for which the localization contribution of the T-fixed locus $\bar{M}_{g, n}\left(\mathbb{P}^{2}, d\right)^{\top}, p_{i}$ to the equivariant Gromov-Witten invariants of $K \mathbb{P}^{2}$ can be written as

$$
\begin{gathered}
\sum_{d=0}^{\infty} Q^{d} \int_{\left[\bar{M}_{g, n}\left(K \mathbb{P}^{2}, d\right)^{\left.\mathrm{\top}, p_{i}\right] \mathrm{vir}}\right.} \psi_{1}^{a_{1}} \cdots \psi_{n}^{a_{n}}= \\
\sum_{k=0}^{\infty} \frac{1}{k !} \int_{\bar{M}_{g, n+k}} \mathrm{H}_{g}^{p_{i}} \psi_{1}^{a_{1}} \cdots \psi_{n}^{a_{n}} \prod_{j=1}^{k} S_{p_{i}}\left(\psi_{n+j}\right) .
\end{gathered}
$$

Here, $\mathrm{H}_{g}^{p_{i}}$ is the standard vertex class,

$$
\frac{e\left(\mathbb{E}_{g}^{*} \otimes T_{p_{i}}\left(\mathbb{P}^{2}\right)\right)}{e\left(T_{p_{i}}\left(\mathbb{P}^{2}\right)\right)} \cdot \frac{e\left(\mathbb{E}_{g}^{*} \otimes\left(-3 \lambda_{i}\right)\right)}{\left(-3 \lambda_{i}\right)},
$$

obtained the Hodge bundle $\mathbb{E}_{g} \rightarrow \bar{M}_{g, n+k}$.

Similarly, the application of the virtual localization formula to the moduli of stable quotients yields classes

$$
F_{p_{i}, k} \in H^{*}\left(\bar{M}_{g, n \mid k}\right) \otimes_{\mathbb{C}} \Lambda_{i}
$$

for which the contribution of $\bar{Q}_{g, n}\left(\mathbb{P}^{2}, d\right)^{T, p_{i}}$ is given by

$$
\begin{gathered}
\sum_{d=0}^{\infty} q^{d} \int_{\left[\bar{Q}_{g, n}\left(K \mathbb{P}^{2}, d\right)^{\top}, p_{i}\right]^{\mathrm{vir}}} \psi_{1}^{a_{1}} \cdots \psi_{n}^{a_{n}}= \\
\sum_{k=0}^{\infty} \frac{q^{k}}{k !} \int_{\bar{M}_{g, n \mid k}} \mathrm{H}_{g}^{p_{i}} \psi_{1}^{a_{1}} \cdots \psi_{n}^{a_{n}} F_{p_{i}, k} .
\end{gathered}
$$

Here $\bar{M}_{g, n \mid k}$ is the moduli space of genus $g$ curves with markings

$$
\left\{p_{1}, \cdots, p_{n}\right\} \cup\left\{\hat{p}_{1} \cdots \hat{p}_{k}\right\} \in C^{\mathrm{ns}} \subset C
$$

satisfying the conditions

(i) the points $p_{i}$ are distinct,

(ii) the points $\hat{p}_{j}$ are distinct from the points $p_{i}$,

with stability given by the ampleness of

$$
\omega_{C}\left(\sum_{i=1}^{m} p_{i}+\epsilon \sum_{j=1}^{k} \hat{p}_{j}\right)
$$


for every strictly positive $\epsilon \in \mathbb{Q}$.

The Hodge class $\mathrm{H}_{g}^{p_{i}}$ is given again by formula (36) using the Hodge bundle

$$
\mathbb{E}_{g} \rightarrow \bar{M}_{g, n \mid k} .
$$

Definition 16. For $\gamma \in H^{*}\left(\bar{M}_{g, n}\right)$, let

$$
\begin{aligned}
\left\langle\left\langle\psi_{1}^{a_{1}}, \ldots, \psi_{n}^{a_{n}} \mid \gamma\right\rangle\right\rangle_{g, n}^{p_{i}, \infty} & =\sum_{k=0}^{\infty} \frac{1}{k !} \int_{\bar{M}_{g, n+k}} \gamma \psi_{1}^{a_{1}} \cdots \psi_{n}^{a_{n}} \prod_{j=1}^{k} S_{p_{i}}\left(\psi_{n+j}\right), \\
\left\langle\left\langle\psi_{1}^{a_{1}}, \ldots, \psi_{n}^{a_{n}} \mid \gamma\right\rangle\right\rangle_{g, n}^{p_{i}, 0+} & =\sum_{k=0}^{\infty} \frac{q^{k}}{k !} \int_{\bar{M}_{g, n \mid k}} \gamma \psi_{1}^{a_{1}} \cdots \psi_{n}^{a_{n}} F_{p_{i}, k} .
\end{aligned}
$$

Proposition 17 (Ciocan-Fontanine, Kim [10]). For $2 g-2+n>0$, we have the wall crossing relation

$$
\left\langle\left\langle\psi_{1}^{a_{1}}, \ldots, \psi_{n}^{a_{n}} \mid \gamma\right\rangle\right\rangle_{g, n}^{p_{i}, \infty}(Q(q))=\left\langle\left\langle\psi_{1}^{a_{1}}, \ldots, \psi_{n}^{a_{n}} \mid \gamma\right\rangle\right\rangle_{g, n}^{p_{i}, 0+}(q)
$$

where $Q(q)$ is the mirror map

$$
Q(q)=\exp \left(I_{1}^{K \mathbb{P}^{2}}(q)\right) .
$$

Proposition 17 is a consequence of [10, Lemma 5.5.1]. The mirror map here is the mirror map for $K \mathbb{P}^{2}$ discussed in Section 0.4. Propositions 13 and 17 together yield

$$
\begin{aligned}
\langle\langle 1, \ldots, 1 \mid \gamma\rangle\rangle_{g, n}^{p_{i}, \infty} & =\mathrm{P}_{g, n}^{0, \ldots, 0, \gamma}\left(\langle\langle 1,1,1\rangle\rangle_{0,3}^{p_{i}, \infty},\langle\langle 1,1,1,1\rangle\rangle_{0,4}^{p_{i}, \infty}, \ldots\right), \\
\langle\langle 1, \ldots, 1 \mid \gamma\rangle\rangle_{g, n}^{p_{i}, 0+} & =\mathrm{P}_{g, n}^{0, \ldots, 0, \gamma}\left(\langle\langle 1,1,1\rangle\rangle_{0,3}^{p_{i}, 0+},\langle\langle 1,1,1,1\rangle\rangle_{0,4}^{p_{i}, 0+}, \ldots\right) .
\end{aligned}
$$

Similarly, using Propositions 14 and 17 , we obtain

$$
\begin{gathered}
\left\langle\left\langle\frac{1}{x_{1}-\psi}, \ldots, \frac{1}{x_{n}-\psi} \mid \gamma\right\rangle\right\rangle_{g, n}^{p_{i}, \infty}= \\
e^{\langle\langle 1,1\rangle\rangle_{0,2}^{p_{i}, \infty}\left(\sum_{i} \frac{1}{x_{i}}\right)} \sum_{a_{1}, \ldots, a_{n}} \frac{\mathrm{P}_{g, n}^{a_{1}, \ldots, a_{n}, \gamma}\left(\langle\langle 1,1,1\rangle\rangle_{0,3}^{p_{i}, \infty},\langle\langle 1,1,1,1\rangle\rangle_{0,4}^{p_{i}, \infty}, \ldots\right)}{x_{1}^{a_{1}+1} \cdots x_{n}^{a_{n}+1}}, \\
(37) \quad\left\langle\left\langle\frac{1}{x_{1}-\psi}, \ldots, \frac{1}{x_{n}-\psi} \mid \gamma\right\rangle\right\rangle_{g, n}^{p_{i}, 0+}= \\
e^{\langle\langle 1,1\rangle\rangle_{0,2}^{p_{i}, 0+}\left(\sum_{i} \frac{1}{x_{i}}\right)} \sum_{a_{1}, \ldots, a_{n}} \frac{\mathrm{P}_{g, n}^{a_{1}, \ldots, a_{n}, \gamma}\left(\langle\langle 1,1,1\rangle\rangle_{0,3}^{p_{i}, 0+},\langle\langle 1,1,1,1\rangle\rangle_{0,4}^{p_{i}, 0+}, \ldots\right)}{x_{1}^{a_{1}+1} \cdots x_{n}^{a_{n}+1}} .
\end{gathered}
$$




\section{Higher GenUS SERIES ON $K \mathbb{P}^{2}$}

5.1. Overview. We apply the localization strategy introduced first by Givental [20, 21, 26] for Gromov-Witten theory to the stable quotient invariants of local $\mathbb{P}^{2}$. The contribution $\operatorname{Cont}_{\Gamma}(q)$ discussed in Section 2 of a graph $\Gamma \in \mathrm{G}_{g}\left(\mathbb{P}^{2}\right)$ can be separated into vertex and edge contributions. We express the vertex and edge contributions in terms of the series $\mathbb{S}_{i}$ and $\mathbb{V}_{i j}$ of Section 3.3 .

5.2. Edge terms. Recall the definition ${ }^{13}$ bf $\mathbb{V}_{i j}$ given in Section 3.3 .

$$
\mathbb{V}_{i j}=\left\langle\left\langle\frac{\phi_{i}}{x-\psi}, \frac{\phi_{j}}{y-\psi}\right\rangle\right\rangle_{0,2}^{0+, 0+}
$$

Let $\overline{\mathbb{V}}_{i j}$ denote the restriction of $\mathbb{V}_{i j}$ to $t=0$. Via formula (17), $\overline{\mathbb{V}}_{i j}$ is a summation of contributions of fixed loci indexed by a graph $\Gamma$ consisting of two vertices connected by a unique edge. Let $w_{1}$ and $w_{2}$ be T-weights. Denote by

$$
\overline{\mathrm{V}}_{i j}^{w_{1}, w_{2}}
$$

the summation of contributions of $\mathrm{T}$-fixed loci with tangent weights precisely $w_{1}$ and $w_{2}$ on the first rational components which exit the vertex components over $p_{i}$ and $p_{j}$.

The series $\overline{\mathbb{V}}_{i j}^{w_{1}, w_{2}}$ includes both vertex and edge contributions. By definition (38) and the virtual localization formula, we find the following relationship between $\overline{\mathbb{V}}_{i j}^{w_{1}, w_{2}}$ and the corresponding pure edge contribution $\mathrm{E}_{i j}^{w_{1}, w_{2}}$,

$$
\begin{aligned}
e_{i} \overline{\mathbb{V}}_{i j}^{w_{1}, w_{2}} e_{j} & =\left\langle\left\langle\frac{1}{w_{1}-\psi}, \frac{1}{x_{1}-\psi}\right\rangle\right\rangle_{0,2}^{p_{i}, 0+} \mathrm{E}_{i j}^{w_{1}, w_{2}}\left\langle\left\langle\frac{1}{w_{2}-\psi}, \frac{1}{x_{2}-\psi}\right\rangle\right\rangle_{0,2}^{p_{j}, 0+} \\
& =\frac{e^{\frac{\langle\langle 1,1\rangle\rangle_{0,2}^{p_{i}, 0+}}{w_{1}}+\frac{\langle\langle 1,1\rangle\rangle_{0,2}^{p_{j}, 0+}}{x_{1}}}}{w_{1}+x_{1}} \mathrm{E}_{i j}^{w_{1}, w_{2}} \frac{e^{\frac{\langle\langle 1,1\rangle\rangle_{0,2}^{p_{i}, 0+}}{w_{2}}+\frac{\langle\langle 1,1\rangle\rangle_{0,2}^{p_{j}, 0+}}{x_{2}}}}{w_{2}+x_{2}} \\
= & \sum_{a_{1}, a_{2}} e^{\frac{\langle\langle 1,1\rangle\rangle_{0,2}^{p_{i}, 0+}}{x_{1}}+\frac{\langle\langle 1,1\rangle\rangle_{0,2}^{p_{i}, 0+}}{w_{1}}} e^{\frac{\langle\langle 1,1\rangle\rangle_{0,2}^{p_{j}, 0+}}{x_{2}}+\frac{\langle\langle 1,1\rangle\rangle_{0,2}^{p_{j}, 0+}}{w_{2}}}(-1)^{a_{1}+a_{2}} \frac{\mathrm{E}_{i j}^{w_{1}, w_{2}}}{w_{1}^{a_{1}} w_{2}^{a_{2}} x_{1}^{a_{1}-1} x_{2}^{a_{2}-1}}
\end{aligned}
$$

After summing over all possible weights, we obtain

$$
e_{i}\left(\overline{\mathbb{V}}_{i j}-\frac{\delta_{i j}}{e_{i}(x+y)}\right) e_{j}=\sum_{w_{1}, w_{2}} e_{i} \overline{\mathbb{V}}_{i j}^{w_{1}, w_{2}} e_{j} .
$$

The above calculations immediately yield the following result.

\footnotetext{
${ }^{13}$ We use the variables $x_{1}$ and $x_{2}$ here instead of $x$ and $y$.
} 
Lemma 18. We have

$$
\begin{gathered}
{\left[e^{-\frac{\langle\langle 1,1\rangle\rangle_{0,2}^{p_{i}, 0+}}{x_{1}}} e^{-\frac{\langle\langle 1,1\rangle\rangle_{0,2}^{p_{j}, 0+}}{x_{2}}} e_{i}\left(\overline{\mathbb{V}}_{i j}-\frac{\delta_{i j}}{e_{i}(x+y)}\right) e_{j}\right]_{x_{1}^{a_{1}-1} x_{2}^{a_{2}-1}}=} \\
\sum_{w_{1}, w_{2}} e^{\frac{\langle\langle 1,1\rangle\rangle_{0,2}^{p_{i}, 0+}}{w_{1}}} e^{\frac{\langle\langle 1,1\rangle\rangle_{0,2}^{p_{j}, 0+}}{w_{2}}}(-1)^{a_{1}+a_{2}} \frac{\mathrm{E}_{i j}^{w_{1}, w_{2}}}{w_{1}^{a_{1}} w_{2}^{a_{2}}} .
\end{gathered}
$$

The notation $[\ldots]_{x_{1}^{a_{1}-1}} x_{2}^{a_{2}-1}$ in Lemma 18 denotes the coefficient of $x_{1}^{a_{1}-1} x_{2}^{a_{2}-1}$ in the series expansion of the argument.

5.3. A simple graph. Before treating the general case, we present the localization formula for a simple graph ${ }^{14}$. Let $\Gamma \in \mathrm{G}_{g}\left(\mathbb{P}^{2}\right)$ consist of two vertices and one edge,

$$
v_{1}, v_{2} \in \Gamma(V), \quad e \in \Gamma(E)
$$

with genus and $\mathrm{T}$-fixed point assignments

$$
\mathrm{g}\left(v_{i}\right)=g_{i}, \quad \mathrm{p}\left(v_{i}\right)=p_{i} .
$$

Let $w_{1}$ and $w_{2}$ be tangent weights at the vertices $p_{1}$ and $p_{2}$ respectively. Denote by $\operatorname{Cont}_{\Gamma, w_{1}, w_{2}}$ the summation of contributions to

$$
\sum_{d>0} q^{d}\left[\bar{Q}_{g}\left(K \mathbb{P}^{2}, d\right)\right]^{\text {vir }}
$$

of T-fixed loci with tangent weights precisely $w_{1}$ and $w_{2}$ on the first rational components which exit the vertex components over $p_{1}$ and $p_{2}$. We can express the localization formula for $(39)$ as

$$
\left\langle\left\langle\frac{1}{w_{1}-\psi} \mid \mathrm{H}_{g_{1}}^{p_{1}}\right\rangle\right\rangle_{g_{1}, 1}^{p_{1}, 0+} \mathrm{E}_{12}^{w_{1}, w_{2}}\left\langle\left\langle\frac{1}{w_{2}-\psi} \mid \mathrm{H}_{g_{2}}^{p_{2}}\right\rangle\right\rangle_{g_{2}, 1}^{p_{2}, 0+}
$$

which equals

$$
\sum_{a_{1}, a_{2}} e^{\frac{\langle\langle 1,1\rangle\rangle_{0,2}^{p_{1}, 0+}}{w_{1}}} \frac{\mathrm{P}\left[\psi^{a_{1}-1} \mid \mathrm{H}_{g_{1}}^{p_{1}}\right]_{g_{1}, 1}^{p_{1}, 0+}}{w_{1}^{a_{1}}} \mathbf{E}_{12}^{w_{1}, w_{2}} e^{\frac{\langle\langle 1,1\rangle\rangle_{0,2}^{p_{2}, 0+}}{w_{2}}} \frac{\mathrm{P}\left[\psi^{a_{2}-1} \mid \mathrm{H}_{g_{2}}^{p_{2}}\right]_{g_{2}, 1}^{p_{2}, 0+}}{w_{2}^{a_{2}}}
$$

where $\mathrm{H}_{g_{i}}^{p_{i}}$ is the Hodge class $(36)$. We have used here the notation

$$
\begin{aligned}
\mathrm{P}\left[\psi_{1}^{k_{1}}, \ldots, \psi_{n}^{k_{n}} \mid \mathrm{H}_{h}^{p_{i}}\right]_{h, n}^{p_{i}, 0+} & = \\
& \mathrm{P}_{h, 1}^{k_{1}, \ldots, k_{n}, \mathrm{H}_{h}^{p_{i}}}\left(\langle\langle 1,1,1\rangle\rangle_{0,3}^{p_{i}, 0+},\langle\langle 1,1,1,1\rangle\rangle_{0,4}^{p_{i}, 0+}, \ldots\right)
\end{aligned}
$$

and applied (37).

\footnotetext{
${ }^{14}$ We follow here the notation of Section 2
} 
After summing over all possible weights $w_{1}, w_{2}$ and applying Lemma 18, we obtain the following result for the full contribution

$$
\operatorname{Cont}_{\Gamma}=\sum_{w_{1}, w_{2}} \operatorname{Cont}_{\Gamma, w_{1}, w_{2}}
$$

of $\Gamma$ to $\sum_{d \geq 0} q^{d}\left[\bar{Q}_{g}\left(K \mathbb{P}^{2}, d\right)\right]^{\text {vir }}$.

Proposition 19. We have

$$
\begin{aligned}
& \operatorname{Cont}_{\Gamma}=\sum_{a_{1}, a_{2}>0} \mathrm{P}\left[\psi^{a_{1}-1} \mid \mathrm{H}_{g_{1}}^{p_{i}}\right]_{g_{1}, 1}^{p_{i}, 0+} \mathrm{P}\left[\psi^{a_{2}-1} \mid \mathrm{H}_{g_{2}}^{p_{j}}\right]_{g_{2}, 1}^{p_{j}, 0+} \\
& \cdot(-1)^{a_{1}+a_{2}}\left[e^{-\frac{\langle\langle 1,1\rangle\rangle_{0,2}^{p_{i}, 0+}}{x_{1}}} e^{-\frac{\langle\langle 1,1\rangle\rangle_{0,2}^{p_{j}, 0+}}{x_{2}}} e_{i}\left(\overline{\mathbb{V}}_{i j}-\frac{\delta_{i j}}{e_{i}\left(x_{1}+x_{2}\right)}\right) e_{j}\right]_{x_{1}^{a_{1}-1} x_{2}^{a_{2}-1}} .
\end{aligned}
$$

5.4. A general graph. We apply the argument of Section 5.3 to obtain a contribution formula for a general graph $\Gamma$.

Let $\Gamma \in \mathrm{G}_{g, 0}\left(\mathbb{P}^{2}\right)$ be a decorated graph as defined in Section 2 , The flags of $\Gamma$ are the half-edge: ${ }^{15}$. Let $\mathrm{F}$ be the set of flags. Let

$$
w: F \rightarrow \operatorname{Hom}\left(T, \mathbb{C}^{*}\right) \otimes_{\mathbb{Z}} \mathbb{Q}
$$

be a fixed assignment of $\mathrm{T}$-weights to each flag.

We first consider the contribution Cont $_{\Gamma, w}$ to

$$
\sum_{d \geq 0} q^{d}\left[\bar{Q}_{g}\left(K \mathbb{P}^{2}, d\right)\right]^{\text {vir }}
$$

of the T-fixed loci associated $\Gamma$ satisfying the following property: the tangent weight on the first rational component corresponding to each $f \in \mathrm{F}$ is exactly given by $\mathrm{w}(f)$. We have

$$
\operatorname{Cont}_{\Gamma, \mathrm{w}}=\frac{1}{|\operatorname{Aut}(\Gamma)|} \sum_{\mathrm{A} \in \mathbb{Z}_{>0}^{\mathrm{F}}} \prod_{v \in \mathrm{V}} \operatorname{Cont}_{\Gamma, \mathrm{w}}^{\mathrm{A}}(v) \prod_{e \in \mathrm{E}} \operatorname{Cont}_{\Gamma, \mathrm{w}}(e) .
$$

The terms on the right side of 40 require definition:

- The sum on the right is over the set $\mathbb{Z}_{>0}^{\mathrm{F}}$ of all maps

$$
A: F \rightarrow \mathbb{Z}_{>0}
$$

corresponding to the sum over $a_{1}, a_{2}$ in Proposition 19 .

\footnotetext{
${ }^{15}$ Flags are either half-edges or markings.
} 
- For $v \in \mathrm{V}$ with $n$ incident flags with $\mathbf{w}$-values $\left(w_{1}, \ldots, w_{n}\right)$ and A-values $\left(a_{1}, a_{2}, \ldots, a_{n}\right)$,

$$
\operatorname{Cont}_{\Gamma, \mathrm{w}}^{\mathrm{A}}(v)=\frac{\mathrm{P}\left[\psi_{1}^{a_{1}-1}, \ldots, \psi_{n}^{a_{n}-1} \mid \mathrm{H}_{\mathrm{g}(v)}^{\mathrm{p}(v)}\right]_{\mathrm{g}(v), n}^{\mathrm{p}(v), 0+}}{w_{1}^{a_{1}} \cdots w_{n}^{a_{n}}} .
$$

- For $e \in \mathbf{E}$ with assignments $\left(\mathbf{p}\left(v_{1}\right), \mathbf{p}\left(v_{2}\right)\right)$ for the two associated vertices $^{16}$ and $w$-values $\left(w_{1}, w_{2}\right)$ for the two associated flags,

$$
\operatorname{Cont}_{\Gamma, \mathrm{w}}(e)=e^{\frac{\langle\langle 1,1\rangle\rangle_{0,2}^{\mathrm{p}\left(v_{1}\right), 0+}}{w_{1}}} e^{\frac{\langle\langle 1,1\rangle\rangle_{0,2}^{\mathrm{p}\left(v_{2}\right), 0+}}{w_{2}}} \mathrm{E}_{\mathbf{p}\left(v_{1}\right), \mathbf{p}\left(v_{2}\right)}^{w_{1}, w_{2}} .
$$

The localization formula then yields (40) just as in the simple case of Section 5.3 .

By summing the contribution (40) of $\Gamma$ over all the weight functions $w$ and applying Lemma 18, we obtain the following result which generalizes Proposition 19 .

Proposition 20. We have

$$
\operatorname{Cont}_{\Gamma}=\frac{1}{|\operatorname{Aut}(\Gamma)|} \sum_{\mathrm{A} \in \mathbb{Z}_{>0}^{\mathrm{F}}} \prod_{v \in \mathrm{V}} \operatorname{Cont}_{\Gamma}^{\mathrm{A}}(v) \prod_{e \in \mathrm{E}} \operatorname{Cont}_{\Gamma}^{\mathrm{A}}(e)
$$

where the vertex and edge contributions with incident flag A-values $\left(a_{1}, \ldots, a_{n}\right)$ and $\left(b_{1}, b_{2}\right)$ respectively are

$$
\begin{aligned}
\operatorname{Cont}_{\Gamma}^{\mathrm{A}}(v) & =\mathrm{P}\left[\psi_{1}^{a_{1}-1}, \ldots, \psi_{n}^{a_{n}-1} \mid \mathrm{H}_{\mathrm{g}(v)}^{\mathrm{p}(v)}\right]_{\mathrm{g}(v), n}^{\mathrm{p}(v), 0+}, \\
\operatorname{Cont}_{\Gamma}^{\mathrm{A}}(e) & =(-1)^{b_{1}+b_{2}}\left[e^{-\frac{\langle\langle 1,1\rangle\rangle_{0,2}^{\mathrm{p}\left(v_{1}\right), 0+}}{x_{1}}} e^{-\frac{\langle\langle 1,1\rangle\rangle_{0,2}^{\mathrm{p}\left(v_{2}\right), 0+}}{x_{2}}} e_{i}\left(\overline{\mathbb{V}}_{i j}-\frac{1}{e_{i}(x+y)}\right) e_{j}\right]_{x_{1}^{b_{1}-1} x_{2}^{b_{2}-1}},
\end{aligned}
$$

where $\mathrm{p}\left(v_{1}\right)=p_{i}$ and $\mathrm{p}\left(v_{2}\right)=p_{j}$ in the second equation.

5.5. Legs. Let $\Gamma \in \mathrm{G}_{g, n}\left(\mathbb{P}^{2}\right)$ be a decorated graph with markings. While no markings are needed to define the stable quotient invariants of $K \mathbb{P}^{2}$, the contributions of decorated graphs with markings will appear in the proof of the holomorphic anomaly equation. The formula for the contribution $\operatorname{Cont}_{\Gamma}\left(H^{k_{1}}, \ldots, H^{k_{n}}\right)$ of $\Gamma$ to

$$
\sum_{d \geq 0} q^{d} \prod_{j=0}^{n} \mathrm{ev}^{*}\left(H^{k_{j}}\right) \cap\left[\bar{Q}_{g, n}\left(K \mathbb{P}^{2}, d\right)\right]^{\mathrm{vir}}
$$

is given by the following result.

\footnotetext{
${ }^{16}$ In case $e$ is self-edge, $v_{1}=v_{2}$.
} 
Proposition 21. We have

$$
\begin{aligned}
& \operatorname{Cont}_{\Gamma}\left(H^{k_{1}}, \ldots, H^{k_{n}}\right)= \\
& \frac{1}{|\operatorname{Aut}(\Gamma)|} \sum_{\mathrm{A} \in \mathbb{Z}_{>0}^{\mathrm{F}}} \prod_{v \in \mathrm{V}} \operatorname{Cont}_{\Gamma}^{\mathrm{A}}(v) \prod_{e \in \mathrm{E}} \operatorname{Cont}_{\Gamma}^{\mathrm{A}}(e) \prod_{l \in \mathrm{L}} \operatorname{Cont}_{\Gamma}^{\mathrm{A}}(l),
\end{aligned}
$$

where the leg contribution is

$$
\operatorname{Cont}_{\Gamma}^{\mathrm{A}}(l)=(-1)^{\mathrm{A}(l)-1}\left[e^{-\frac{\langle\langle 1,1\rangle\rangle_{0,2}^{\mathrm{p}(l), 0+}}{z}} \overline{\mathbb{S}}_{\mathrm{p}(l)}\left(H^{k_{l}}\right)\right]_{z^{\mathrm{A}(l)-1}} .
$$

The vertex and edge contributions are same as before.

The proof of Proposition 21 follows the vertex and edge analysis. We leave the details as an exercise for the reader. The parallel statement for Gromov-Witten theory can be found in [20, 21, 26].

\section{Vertices, EDGES, AND LEGS}

6.1. Overview. Using the results of Givental [20, 21, 26] combined with wall-crossing [10], we calculate here the vertex and edge contributions in terms of the function $R_{k}$ of Section 3.4.4.

6.2. Calculations in genus $\mathbf{0}$. We follow the notation introduced in Section 4.1. Recall the series

$$
T(c)=t_{0}+t_{1} c+t_{2} c^{2}+\ldots .
$$

Proposition 22. (Givental [20, 21, 26]) For $n \geq 3$, we have

$$
\begin{aligned}
& \langle\langle 1, \ldots, 1\rangle\rangle_{0, n}^{p_{i}, \infty}= \\
& \left.\quad\left(\sum_{k \geq 0} \frac{1}{k !} \int_{\bar{M}_{0, n+k}} T\left(\psi_{n+1}\right) \cdots T\left(\psi_{n+k}\right)\right)\right|_{t_{0}=0, t_{1}=0, t_{j \geq 2}=(-1)^{j}} \frac{Q_{j-1}}{\lambda_{i}^{j-1}}
\end{aligned}
$$

where the functions $Q_{l}$ are defined by

$$
\overline{\mathbb{S}}_{i}^{\infty}(1)=e_{i}\left\langle\left\langle\frac{\phi_{i}}{z-\psi}, 1\right\rangle\right\rangle_{0,2}^{p_{i}, \infty}=e^{\frac{\langle\langle 1,1\rangle\rangle_{0,2}^{p_{i}, \infty}}{z}}\left(\sum_{l=0}^{\infty} Q_{l}\left(\frac{z}{\lambda_{i}}\right)^{l}\right) .
$$

The existence of the above asymptotic expansion of $\overline{\mathbb{S}}_{i}^{\infty}(1)$ can also be proven by the argument of [7, Theorem 5.4.1]. Similarly, we have an asymptotic expansion of $\overline{\mathbb{S}}_{i}(1)$,

$$
\overline{\mathbb{S}}_{i}(1)=e^{\frac{\langle\langle 1,1\rangle\rangle_{0,2}^{p_{i}, 0+}}{z}}\left(\sum_{l=0}^{\infty} R_{l}\left(\frac{z}{\lambda_{i}}\right)^{l}\right) .
$$


By (30), we have

$$
\langle\langle 1,1\rangle\rangle_{0,2}^{p_{i}, 0+}=\mu \lambda_{i}
$$

After applying the wall-crossing result of Proposition 17, we obtain

$$
\begin{aligned}
\langle\langle 1, \ldots, 1\rangle\rangle_{0, n}^{p_{i}, \infty}(Q(q)) & =\langle\langle 1, \ldots, 1\rangle\rangle_{0, n}^{p_{i}, 0+}(q), \\
\overline{\mathbb{S}}_{i}^{\infty}(1)(Q(q)) & =\overline{\mathbb{S}}_{i}(1)(q),
\end{aligned}
$$

where $Q(q)$ is mirror map for $K \mathbb{P}^{2}$ as before. By comparing asymptotic expansions of $\bar{S}_{i}^{\infty}(1)$ and $\overline{\mathbb{S}}_{i}(1)$, we get a wall-crossing relation between $Q_{l}$ and $R_{l}$,

$$
Q_{l}(Q(q))=R_{l}(q)
$$

We have proven the following result.

Proposition 23. For $n \geq 3$, we have

$$
\begin{aligned}
& \langle\langle 1, \ldots, 1\rangle\rangle_{0, n}^{p_{i}, 0+}= \\
& \left.\quad\left(\sum_{k \geq 0} \frac{1}{k !} \int_{\bar{M}_{0, n+k}} T\left(\psi_{n+1}\right) \cdots T\left(\psi_{n+k}\right)\right)\right|_{t_{0}=0, t_{1}=0, t_{j \geq 2}=(-1)^{j} \frac{R_{j-1}}{\lambda_{i}^{j-1}}} .
\end{aligned}
$$

Proposition 23 immediately implies the evaluation

$$
\langle\langle 1,1,1\rangle\rangle_{0,3}^{p_{i}, 0+}=1 .
$$

Another simple consequence of Proposition 23 is the following basic property.

Corollary 24. For $n \geq 3$, we have $\langle\langle 1, \ldots, 1\rangle\rangle_{0, n}^{p_{i}, 0+} \in \mathbb{C}\left[R_{1}, R_{2}, \ldots\right]\left[\lambda_{i}^{-1}\right]$.

6.3. Vertex and edge analysis. By Proposition 20, we have decomposition of the contribution to $\Gamma \in \mathrm{G}_{g}\left(\mathbb{P}^{2}\right)$ to the stable quotient theory of $K \mathbb{P}^{2}$ into vertex terms and edge terms

$$
\operatorname{Cont}_{\Gamma}=\frac{1}{|\operatorname{Aut}(\Gamma)|} \sum_{\mathrm{A} \in \mathbb{Z}_{>0}^{\mathrm{F}}} \prod_{v \in \mathrm{V}} \operatorname{Cont}_{\Gamma}^{\mathrm{A}}(v) \prod_{e \in \mathrm{E}} \operatorname{Cont}_{\Gamma}^{\mathrm{A}}(e) .
$$

Lemma 25. We have $\operatorname{Cont}_{\Gamma}^{\mathrm{A}}(v) \in \mathbb{C}\left(\lambda_{0}, \lambda_{1}, \lambda_{2}\right)\left[L^{ \pm 1}\right]$.

Proof. By Proposition 20,

$$
\operatorname{Cont}_{\Gamma}^{\mathrm{A}}(v)=\mathrm{P}\left[\psi_{1}^{a_{1}-1}, \ldots, \psi_{n}^{a_{n}-1} \mid \mathrm{H}_{\mathrm{g}(v)}^{\mathrm{p}(v)}\right]_{\mathrm{g}(v), n}^{\mathrm{p}(v), 0+} .
$$

The right side of the above formula is a polynomial in the variables

$$
\frac{1}{\langle\langle 1,1,1\rangle\rangle_{0,3}^{\mathrm{p}(v), 0+}} \quad \text { and } \quad\left\{\left.\langle\langle 1, \ldots, 1\rangle\rangle_{0, n}^{\mathrm{p}(v), 0+}\right|_{t_{0}=0}\right\}_{n \geq 4}
$$


with coefficients in $\mathbb{C}\left(\lambda_{0}, \lambda_{1}, \lambda_{2}\right)$. The Lemma then follows from the evaluation (41), Corollary 24, and Proposition 7 .

Let $e \in \mathrm{E}$ be an edge connecting the T-fixed points $p_{i}, p_{j} \in \mathbb{P}^{2}$. Let the $\mathrm{A}$-values of the respective half-edges be $(k, l)$.

Lemma 26. We have $\operatorname{Cont}_{\Gamma}^{\mathrm{A}}(e) \in \mathbb{C}\left(\lambda_{0}, \lambda_{1}, \lambda_{2}\right)\left[L^{ \pm 1}, X\right]$ and

- the degree of $\operatorname{Cont}_{\Gamma}^{\mathrm{A}}(e)$ with respect to $X$ is 1 ,

- the coefficient of $X$ in $\operatorname{Cont}_{\Gamma}^{\mathrm{A}}(e)$ is

$$
(-1)^{k+l} \frac{3 R_{1 k-1} R_{1-1-1}}{L \lambda_{i}^{k-2} \lambda_{j}^{l-2}}
$$

Proof. By Proposition 20,

$$
\operatorname{Cont}_{\Gamma}^{\mathrm{A}}(e)=(-1)^{k+l}\left[e^{-\frac{\mu \lambda_{i}}{x}-\frac{\mu \lambda_{j}}{y}} e_{i}\left(\overline{\mathbb{V}}_{i j}-\frac{\delta_{i j}}{e_{i}(x+y)}\right) e_{j}\right]_{x^{k-1} y^{l-1}} .
$$

Using also the equation

$$
e_{i} \overline{\mathbb{V}}_{i j}(x, y) e_{j}=\frac{\left.\left.\sum_{r=0}^{2} \overline{\mathbb{S}}_{i}\left(\phi_{r}\right)\right|_{z=x} \overline{\mathbb{S}}_{j}\left(\phi^{r}\right)\right|_{z=y}}{x+y}
$$

we write $\operatorname{Cont}_{\Gamma}^{\mathrm{A}}(e)$ as

$$
\left[\left.\left.(-1)^{k+l} e^{-\frac{\mu \lambda_{i}}{x}-\frac{\mu \lambda_{j}}{y}} \sum_{r=0}^{2} \overline{\mathbb{S}}_{i}\left(\phi_{r}\right)\right|_{z=x} \overline{\mathbb{S}}_{j}\left(\phi^{r}\right)\right|_{z=y}\right]_{x^{k} y^{l-1}-x^{k+1} y^{l-2}+\ldots+(-1)^{k-1} x^{k+l-1}}
$$

where the subscript signifies a (signed) sum of the respective coefficients. If we substitute the asymptotic expansions (30) for

$$
\overline{\mathbb{S}}_{i}(1), \overline{\mathbb{S}}_{i}(H), \quad \overline{\mathbb{S}}_{i}\left(H^{2}\right)
$$

in the above expression, the Lemma follows from Proposition 7 and Lemma 8.

6.4. Legs. Using the contribution formula of Proposition 21,

$$
\operatorname{Cont}_{\Gamma}^{\mathrm{A}}(l)=(-1)^{\mathrm{A}(l)-1}\left[e^{-\frac{\langle\langle 1,1\rangle\rangle_{0,2}^{\mathrm{p}(l), 0+}}{z}} \overline{\mathbb{S}}_{\mathrm{p}(l)}\left(H^{k_{l}}\right)\right]_{z^{\mathrm{A}(l)-1}},
$$

we easily conclude

- when the insertion at the marking $l$ is $H^{0}$,

$$
\operatorname{Cont}_{\Gamma}^{\mathrm{A}}(l) \in \mathbb{C}\left(\lambda_{0}, \lambda_{1}, \lambda_{2}\right)\left[L^{ \pm 1}\right],
$$

- when the insertion at the marking $l$ is $H^{1}$,

$$
C_{1} \cdot \operatorname{Cont}_{\Gamma}^{\mathrm{A}}(l) \in \mathbb{C}\left(\lambda_{0}, \lambda_{1}, \lambda_{2}\right)\left[L^{ \pm 1}\right],
$$


- when the insertion at the marking $l$ is $H^{2}$,

$$
C_{1} C_{2} \cdot \operatorname{Cont}_{\Gamma}^{\mathrm{A}}(l) \in \mathbb{C}\left(\lambda_{0}, \lambda_{1}, \lambda_{2}\right)\left[L^{ \pm 1}, X\right] .
$$

\section{Holomorphic ANOMAly FOR $K \mathbb{P}^{2}$}

7.1. Proof of Theorem 1. By definition, we have

$$
A_{2}(q)=\frac{1}{L^{3}}\left(3 X+1-\frac{L^{3}}{2}\right) \text {. }
$$

Hence, statement (i),

$$
\mathcal{F}_{g}^{\mathrm{SQ}}(q) \in \mathbb{C}\left[L^{ \pm 1}\right]\left[A_{2}\right],
$$

follows from Proposition 20 and Lemmas 25-26. Statement (ii), $\mathcal{F}_{g}^{\mathrm{SQ}}$ has at most degree $3 g-3$ with respect to $A_{2}$, holds since a stable graph of genus $g$ has at most $3 g-3$ edges. Since

$$
\frac{\partial}{\partial T}=\frac{q}{C_{1}} \frac{\partial}{\partial q}
$$

statement (iii),

$$
\frac{\partial^{k} \mathcal{F}_{g}^{\mathrm{SQ}}}{\partial T^{k}}(q) \in \mathbb{C}\left[L^{ \pm 1}\right]\left[A_{2}\right]\left[C_{1}^{-1}\right]
$$

follows since the ring

$$
\mathbb{C}\left[L^{ \pm 1}\right]\left[A_{2}\right]=\mathbb{C}\left[L^{ \pm 1}\right][X]
$$

is closed under the action of the differential operator

$$
\mathrm{D}=q \frac{\partial}{\partial q}
$$

by (32). The degree of $C_{1}^{-1}$ in 43 is 1 which yields statement (iv).

7.2. Proof of Theorem 2. Let $\Gamma \in \mathrm{G}_{g}\left(\mathbb{P}^{2}\right)$ be a decorated graph. Let us fix an edge $f \in \mathrm{E}(\Gamma)$ :

- if $\Gamma$ is connected after deleting $f$, denote the resulting graph by

$$
\Gamma_{f}^{0} \in \mathrm{G}_{g-1,2}\left(\mathbb{P}^{2}\right),
$$

$\bullet$ if $\Gamma$ is disconnected after deleting $f$, denote the resulting two graphs by

$$
\Gamma_{f}^{1} \in \mathrm{G}_{g_{1}, 1}\left(\mathbb{P}^{2}\right) \quad \text { and } \quad \Gamma_{f}^{2} \in \mathrm{G}_{g_{2}, 1}\left(\mathbb{P}^{2}\right)
$$

where $g=g_{1}+g_{2}$. 
There is no canonical order for the 2 new markings. We will always sum over the 2 labellings. So more precisely, the graph $\Gamma_{f}^{0}$ in case • should be viewed as sum of 2 graphs

$$
\Gamma_{f,(1,2)}^{0}+\Gamma_{f,(2,1)}^{0} \text {. }
$$

Similarly, in case $\bullet \bullet$, we will sum over the ordering of $g_{1}$ and $g_{2}$. As usually, the summation will be later compensated by a factor of $\frac{1}{2}$ in the formulas.

By Proposition 20, we have the following formula for the contribution of the graph $\Gamma$ to the stable quotient theory of $K \mathbb{P}^{2}$,

$$
\operatorname{Cont}_{\Gamma}=\frac{1}{|\operatorname{Aut}(\Gamma)|} \sum_{\mathrm{A} \in \mathbb{Z}_{\geq 0}^{\mathrm{F}}} \prod_{v \in \mathrm{V}} \operatorname{Cont}_{\Gamma}^{\mathrm{A}}(v) \prod_{e \in \mathrm{E}} \operatorname{Cont}_{\Gamma}^{\mathrm{A}}(e) .
$$

Let $f$ connect the $\mathrm{T}$-fixed points $p_{i}, p_{j} \in \mathbb{P}^{2}$. Let the A-values of the respective half-edges be $(k, l)$. By Lemma 26, we have

$$
\frac{\partial \operatorname{Cont}_{\Gamma}^{\mathrm{A}}(f)}{\partial X}=(-1)^{k+l} \frac{3 R_{1 k-1} R_{1 l-1}}{L \lambda_{i}^{k-2} \lambda_{j}^{l-2}} .
$$

- If $\Gamma$ is connected after deleting $f$, we have

$$
\begin{aligned}
\frac{1}{|\operatorname{Aut}(\Gamma)|} \sum_{\mathrm{A} \in \mathbb{Z}_{\geq 0}^{\mathrm{F}}}\left(\frac{L^{3}}{3 C_{1}^{2}}\right) \frac{\partial \operatorname{Cont}_{\Gamma}^{\mathrm{A}}(f)}{\partial X} \prod_{v \in \mathrm{V}} \operatorname{Cont}_{\Gamma}^{\mathrm{A}}(v) & \prod_{e \in \mathrm{E}, e \neq f} \operatorname{Cont}_{\Gamma}^{\mathrm{A}}(e) \\
& =\frac{1}{2} \operatorname{Cont}_{\Gamma_{f}^{0}}(H, H) .
\end{aligned}
$$

The derivation is simply by using (44) on the left and Proposition 21 on the right.

$\bullet$ If $\Gamma$ is disconnected after deleting $f$, we obtain

$$
\begin{aligned}
& \frac{1}{|\operatorname{Aut}(\Gamma)|} \sum_{\mathrm{A} \in \mathbb{Z}_{\geq 0}^{\mathrm{F}}}\left(\frac{L^{3}}{3 C_{1}^{2}}\right) \frac{\partial \operatorname{Cont}_{\Gamma}^{\mathrm{A}}(f)}{\partial X} \prod_{v \in \mathrm{V}} \operatorname{Cont}_{\Gamma}^{\mathrm{A}}(v) \prod_{e \in \mathrm{E}, e \neq f} \operatorname{Cont}_{\Gamma}^{\mathrm{A}}(e) \\
& =\frac{1}{2} \operatorname{Cont}_{\Gamma_{f}^{1}}(H) \operatorname{Cont}_{\Gamma_{f}^{2}}(H)
\end{aligned}
$$

by the same method.

By combining the above two equations for all the edges of all the graphs $\Gamma \in \mathrm{G}_{g}\left(\mathbb{P}^{2}, d\right)$ and using the vanishing

$$
\frac{\partial \operatorname{Cont}_{\Gamma}^{\mathrm{A}}(v)}{\partial X}=0
$$


of Lemma 25, we obtain

$$
\left(\frac{L^{3}}{3 C_{1}^{2}}\right) \frac{\partial}{\partial X}\langle\rangle_{g, 0}^{\mathrm{SQ}}=\frac{1}{2} \sum_{i=1}^{g-1}\langle H\rangle_{g-i, 1}^{\mathrm{SQ}}\langle H\rangle_{i, 1}^{\mathrm{SQ}}+\frac{1}{2}\langle H, H\rangle_{g-1,2}^{\mathrm{SQ}}
$$

We have followed here the notation of Section 0.4. The equality 45 holds in the ring $\mathbb{C}\left[L^{ \pm}\right]\left[A_{2}, C_{1}^{-1}\right]$.

Since $A_{2}=\frac{1}{L^{3}}\left(3 X+1-\frac{L^{3}}{2}\right)$ and \langle\rangle$_{g, 0}^{\mathrm{SQ}}=\mathcal{F}_{g}^{\mathrm{SQ}}$, the left side of 45 is, by the chain rule,

$$
\frac{1}{C_{1}^{2}} \frac{\partial \mathcal{F}_{g}^{\mathrm{SQ}}}{\partial A_{2}} \in \mathbb{C}\left[L^{ \pm}\right]\left[A_{2}, C_{1}^{-1}\right] .
$$

On the right side of (45), we have

$$
\langle H\rangle_{g-i, 1}^{\mathrm{SQ}}=\mathcal{F}_{g-i, 1}^{\mathrm{SQ}}(q)=\mathcal{F}_{g-i, 1}^{\mathrm{GW}}(Q(q)),
$$

where the first equality is by definition and the second is by wallcrossing (7). Then,

$$
\mathcal{F}_{g-i, 1}^{\mathrm{GW}}(Q(q))=\frac{\partial \mathcal{F}_{g-i}^{\mathrm{GW}}}{\partial T}(Q(q))=\frac{\partial \mathcal{F}_{g-i}^{\mathrm{SQ}}}{\partial T}(q)
$$

where the first equality is by the divisor equation in Gromov-Witten theory and the second is again by wall-crossing (7), so we conclude

$$
\langle H\rangle_{g-i, 1}^{\mathrm{SQ}}=\frac{\partial \mathcal{F}_{g-i}^{\mathrm{SQ}}}{\partial T}(q) \in \mathbb{C}[[q]] .
$$

Similarly, we obtain

$$
\begin{aligned}
\langle H\rangle_{i, 1}^{\mathrm{SQ}} & =\frac{\partial \mathcal{F}_{i}^{\mathrm{SQ}}}{\partial T}(q) \in \mathbb{C}[[q]], \\
\langle H, H\rangle_{g-1,2}^{\mathrm{SQ}} & =\frac{\partial^{2} \mathcal{F}_{g-1}^{\mathrm{SQ}}}{\partial T^{2}}(q) \in \mathbb{C}[[q]] .
\end{aligned}
$$

Together, the above equations transform 45) into exactly the holomorphic anomaly equation of Theorem 2 ,

$$
\frac{1}{C_{1}^{2}} \frac{\partial \mathcal{F}_{g}^{\mathrm{SQ}}}{\partial A_{2}}(q)=\frac{1}{2} \sum_{i=1}^{g-1} \frac{\partial \mathcal{F}_{g-i}^{\mathrm{SQ}}}{\partial T}(q) \frac{\partial \mathcal{F}_{i}^{\mathrm{SQ}}}{\partial T}(q)+\frac{1}{2} \frac{\partial^{2} \mathcal{F}_{g-1}^{\mathrm{SQ}}}{\partial T^{2}}(q)
$$

as an equality in $\mathbb{C}[[q]]$.

The series $L$ and $A_{2}$ are expected to be algebraically independent. Since we do not have a proof of the independence, to lift holomorphic anomaly equation to the equality

$$
\frac{1}{C_{1}^{2}} \frac{\partial \mathcal{F}_{g}^{\mathrm{SQ}}}{\partial A_{2}}=\frac{1}{2} \sum_{i=1}^{g-1} \frac{\partial \mathcal{F}_{g-i}^{\mathrm{SQ}}}{\partial T} \frac{\partial \mathcal{F}_{i}^{\mathrm{SQ}}}{\partial T}+\frac{1}{2} \frac{\partial^{2} \mathcal{F}_{g-1}^{\mathrm{SQ}}}{\partial T^{2}}
$$


STABLE QUOTIENTS AND THE HOLOMORPHIC ANOMALY EQUATION 41 in the ring $\mathbb{C}\left[L^{ \pm 1}\right]\left[A_{2}, C_{1}^{-1}\right]$, we must prove the equalities

$$
\begin{gathered}
\langle H\rangle_{g-i, 1}^{\mathrm{SQ}}=\frac{\partial \mathcal{F}_{g-i}^{\mathrm{SQ}}}{\partial T}, \quad\langle H\rangle_{i, 1}^{\mathrm{SQ}}=\frac{\partial \mathcal{F}_{i}^{\mathrm{SQ}}}{\partial T}, \\
\langle H, H\rangle_{g-1,2}^{\mathrm{SQ}}=\frac{\partial^{2} \mathcal{F}_{g-1}^{\mathrm{SQ}}}{\partial T^{2}}
\end{gathered}
$$

in the ring $\mathbb{C}\left[L^{ \pm}\right]\left[A_{2}, C_{1}^{-1}\right]$. The lifting will be proven in Section 7.3 below.

We do not study the genus 1 unpointed series $\mathcal{F}_{1}^{\mathrm{SQ}}(q)$ in the paper, so we take

$$
\begin{aligned}
\langle H\rangle_{1,1}^{\mathrm{SQ}} & =\frac{\partial \mathcal{F}_{1}^{\mathrm{SQ}}}{\partial T}, \\
\langle H, H\rangle_{1,2}^{\mathrm{SQ}} & =\frac{\partial^{2} \mathcal{F}_{1}^{\mathrm{SQ}}}{\partial T^{2}} .
\end{aligned}
$$

as definitions of the right side in the genus 1 case. There is no difficulty in calculating these series explicitly using Proposition 21.

$$
\begin{aligned}
\frac{\partial \mathcal{F}_{1}^{\mathrm{SQ}}}{\partial T} & =-\frac{1}{6 C_{1}} L^{3} A_{2}, \\
\frac{\partial^{2} \mathcal{F}_{1}^{\mathrm{SQ}}}{\partial T^{2}} & =\frac{1}{C_{1}} \mathrm{D}\left(-\frac{1}{6 C_{1}} L^{3} A_{2}\right) .
\end{aligned}
$$

7.3. Lifting. We write the first two equalities in (46) together as

$$
\langle H\rangle_{h, 1}^{\mathrm{SQ}}=\frac{\partial \mathcal{F}_{h}^{\mathrm{SQ}}}{\partial T} .
$$

The formula of Proposition 21 for the left side of (47) is a summation over graphs $\Gamma \in \mathrm{G}_{h, 1}\left(\mathbb{P}^{2}\right)$. Stabilization yields canonical map,

$$
\mathrm{G}_{h, 1}\left(\mathbb{P}^{2}\right) \rightarrow \mathrm{G}_{h, 0}\left(\mathbb{P}^{2}\right), \quad \Gamma \mapsto \widetilde{\Gamma},
$$

obtained by forgetting the marking 1 .

- If the marking 1 is carried by a vertex $v$ which is stable without the marking, $\widetilde{\Gamma}$ is simply obtained by removing the marking. The marking falls to the corresponding vertex $v$ of $\widetilde{\Gamma}$.

- If the marking 1 is carried by a vertex $v$ which is unstable without the marking, then $v$ is contracted in the stabilization. The marking 1 falls to a unique edge of $\widetilde{\Gamma}$.

For a fixed edge $\tilde{f}$ of $\widetilde{\Gamma}$ there are exactly 3 graphs of $G_{h, 1}(\mathbb{P})$ for which the marking 1 falls to $\widetilde{f}$. These come from the 3 possible $\mathrm{p}$ values of the contracted vertex. 
If we start with an edge $\tilde{f}$ of $\widetilde{\Gamma} \in \mathrm{G}_{h, 0}\left(\mathbb{P}^{2}\right)$ connecting ${ }^{17}$ two vertices $v$ and $v^{\prime}$, there are 5 associated graphs in $\mathrm{G}_{h, 1}\left(\mathbb{P}^{2}\right)$ :

- let $\Gamma_{v}^{\tilde{f}}, \Gamma_{v^{\prime}}^{\tilde{f}} \in \mathrm{G}_{h, 1}\left(\mathbb{P}^{2}\right)$ be the graphs where the marking 1 falls to the respective vertices of $\widetilde{\Gamma}$,

- let $\Gamma_{1}^{\widetilde{f}}, \Gamma_{2}^{\widetilde{f}}, \Gamma_{3}^{\widetilde{f}} \in \mathrm{G}_{h, 1}\left(\mathbb{P}^{2}\right)$ be the three graphs where the marking 1 falls to the edge $\tilde{f}$ of $\widetilde{\Gamma}$.

The right side of (47) may also be written as a summation over graphs $\Gamma \in G_{h, 1}\left(\mathbb{P}^{2}\right)$. The formula of Proposition 21 for $\mathcal{F}_{h}^{\mathrm{SQ}}$ is a summation over graphs

$$
\widetilde{\Gamma} \in \mathrm{G}_{h, 0}\left(\mathbb{P}^{2}\right) .
$$

We then view the action of the derivative $\frac{\partial}{\partial T}$ as producing the marking 1. If the derivative acts on a vertex contribution of $\widetilde{\Gamma}$, the marking is distributed to that vertex. If the derivative acts on an edge $\widetilde{f}$ contribution, we view the differentiation as accounting for the sum of the 3 graphs

$$
\Gamma_{1}^{\tilde{f}}, \Gamma_{2}^{\tilde{f}}, \Gamma_{3}^{\tilde{f}} \in \mathrm{G}_{h, 1}\left(\mathbb{P}^{2}\right) .
$$

We now apply Proposition 21 via the above analysis to the difference

$$
\frac{\partial \mathcal{F}_{h}^{\mathrm{SQ}}}{\partial T}-\langle H\rangle_{h, 1}^{\mathrm{SQ}}
$$

The result is a summation of contributions corresponding to graphs $\Gamma \in \mathrm{G}_{h, 1}\left(\mathbb{P}^{2}\right)$ where all triples 48$)$ are considered contributing together.

For graphs $\Gamma \in \mathrm{G}_{h, 1}\left(\mathbb{P}^{2}\right)$ for which the marking 1 falls to a vertex $v$ in $\widetilde{\Gamma}$, we will distribute the vertex contribution naturally to the incident edges of $v \in \widetilde{\Gamma}$ by the following method. Proposition 23 and the string equation yield a local version of divisor equation in the ring $\mathbb{C}\left[L^{ \pm 1}\right]\left[A_{2}, C_{1}^{-1}\right]$. For $\gamma \in H^{*}\left(\bar{M}_{g, n}\right)$,

$$
\begin{aligned}
& \frac{\partial}{\partial T} \mathrm{P}\left[\psi_{1}^{a_{1}}, \ldots, \psi_{n}^{a_{n}} \mid \gamma\right]_{h, n}^{\mathrm{p}(v), 0+}= \\
& \sum_{k \geq 0} \mathrm{P}\left[\psi_{1}^{a_{1}}, \ldots, \psi_{n}^{a_{n}}, \psi_{n+1}^{k} \mid \gamma\right]_{h, n+1}^{\mathrm{p}(v), 0+} \frac{(-1)^{k} \lambda_{\mathrm{p}(v)}^{1-k} L R_{1 k}}{C_{1}} \\
& \quad-\sum_{j=0}^{n} \mathrm{P}\left[\psi_{1}^{a_{1}}, \ldots, \psi_{1}^{a_{j}-1}, \ldots, \psi_{n}^{a_{n}} \mid \gamma\right]_{h, n}^{\mathrm{p}(v), 0+} \frac{\lambda_{\mathrm{p}(v)} L}{C_{1}}
\end{aligned}
$$

\footnotetext{
${ }^{17}$ The analysis of the self edge case is identical and left to the reader.
} 
While Proposition 23 was stated only for genus 0 invariants without $\psi$ insertions, the same result holds for all genera $h$ with $\psi$ by exactly the same argument,

$$
\begin{aligned}
& \text { (51) } \mathrm{P}\left[\psi_{1}^{a_{1}}, \ldots, \psi_{n}^{a_{n}} \mid \gamma\right]_{h, n}^{\mathrm{p}(v), 0+}= \\
& \left.\left(\sum_{k \geq 0} \frac{1}{k !} \int_{\bar{M}_{h, n+k}} \psi_{1}^{a_{1}} \cdots \psi_{n}^{a_{n}} \cdot \gamma \cdot T\left(\psi_{n+1}\right) \cdots T\left(\psi_{n+k}\right)\right)\right|_{t_{0}=0, t_{1}=0, t_{j \geq 2}=(-1)^{j}} \frac{R_{j-1}}{\lambda_{i}^{j-1}}
\end{aligned}
$$

By Proposition 21 with $(50)$, the A-valued contribution ${ }^{18}$ to

$$
\frac{\partial \mathcal{F}_{h}^{\mathrm{SQ}}}{\partial T}-\langle H\rangle_{h, 1}^{\mathrm{SQ}}
$$

of the vertex $v$ is

$$
-\sum_{j=1}^{n} \mathrm{P}\left[\psi_{1}^{a_{1}}, \ldots, \psi_{j}^{a_{j}-1}, \ldots, \psi_{n}^{a_{n}} \mid \mathrm{H}_{\mathrm{g}(v)}^{\mathrm{p}(v)}\right]_{\mathrm{g}(v), n}^{\mathrm{p}(v), 0+} \frac{\lambda_{\mathrm{p}(v)} L}{C_{1}} .
$$

Here, we have used also the following equation for the $H$ insertion on the leg $l$ at the vertex $v$ :

$$
\begin{aligned}
\operatorname{Cont}_{\Gamma}^{\mathrm{A}}(l) & =(-1)^{\mathrm{A}(l)-1}\left[e^{-\frac{\langle\langle 1,1\rangle\rangle_{0,2}^{\mathrm{p}(l), 0+}}{z}} \overline{\mathrm{S}}_{\mathrm{p}(l)}(H)\right]_{z^{\mathrm{A}(l)-1}} \\
& =(-1)^{\mathrm{A}(l)-1} \frac{\lambda_{\mathrm{p}(v)}^{-\mathrm{A}(l)} L R_{1} \mathrm{~A}(l)-1}{C_{1}} .
\end{aligned}
$$

The location of $\psi_{j}$ with exponent $a_{j}-1$ exactly tell us to which edge we distribute.

After applying Proposition 21 to

$$
\frac{\partial \mathcal{F}_{h}^{\mathrm{SQ}}}{\partial T}-\langle H\rangle_{h, 1}^{\mathrm{SQ}}
$$

with above vertex term distribution, the sum of the contributions of the 5 graphs

$$
\Gamma_{v}^{\tilde{f}}, \Gamma_{v^{\prime}}^{\tilde{f}}, \Gamma_{1}^{\tilde{f}}, \Gamma_{2}^{\tilde{f}}, \Gamma_{3}^{\tilde{f}}
$$

\footnotetext{
${ }^{18}$ For $\sum_{i=1}^{n} a_{i}=3 g-3+n+1-r$, where $r$ is degree of $\gamma \in H^{*}\left(\bar{M}_{g, n}\right)$, we use the following equation which can be easily checked by (51) and the string equation,

$$
\mathrm{P}\left[\psi_{1}^{a_{1}}, \ldots, \psi_{n}^{a_{n}}, 1 \mid \gamma\right]_{\mathrm{g}(v), n+1}^{\mathrm{p}(v), 0+}=\sum_{j=1}^{n} \mathrm{P}\left[\psi_{1}^{a_{1}}, \ldots, \psi_{j}^{a_{j}-1}, \ldots, \psi_{n}^{a_{n}} \mid \gamma\right]_{\mathrm{g}(v), n}^{\mathrm{p}(v), 0+} \text {. }
$$
}


related to the edge $\widetilde{f}$ of $\widetilde{\Gamma}$ can be written as:

$$
\begin{aligned}
& -\frac{1}{L} \mathrm{D}\left[e^{-\frac{\mu \lambda_{i}}{x}-\frac{\mu \lambda_{j}}{y}} e_{i} \overline{\mathbb{V}}_{i j}(x, y) e_{j}\right]_{x^{k} y^{l}}+ \\
& \sum_{\alpha=0}^{2}\left[e^{-\frac{\mu \lambda_{i}}{x}-\frac{\mu \lambda_{\alpha}}{z}} e_{i} \overline{\mathbb{V}}_{i \alpha}(x, z) e_{\alpha}\right]_{x^{k}}\left[e^{-\frac{\mu \lambda_{\alpha}}{z}-\frac{\mu \lambda_{j}}{y}} e_{\alpha} \overline{\mathbb{V}}_{\alpha j}(z, y) e_{j}\right]_{y^{l}} \\
& -\left[e^{-\frac{\mu \lambda_{i}}{x}-\frac{\mu \lambda_{j}}{y}} e_{i} \overline{\mathbb{V}}_{i j}(x, y) e_{j}\right]_{x^{k+1} y^{l}}-\left[e^{-\frac{\mu \lambda_{i}}{x}-\frac{\mu \lambda_{j}}{y}} e_{i} \overline{\mathbb{V}}_{i j}(x, y) e_{j}\right]_{x^{k} y^{l+1}} .
\end{aligned}
$$

The first two terms come from sum of the triple $\Gamma_{1}^{\widetilde{f}}, \Gamma_{2}^{\widetilde{f}}, \Gamma_{3}^{\widetilde{f}}$, and last two terms come from $\Gamma_{v}^{\widetilde{f}}, \Gamma_{v^{\prime}}^{\widetilde{f}}$. The vanishing of above sum in the ring $\mathbb{C}\left[L^{ \pm 1}\right]\left[A_{2}, C_{1}^{-1}\right]$ is easily obtained using Lemma 9 (including relation (32)).

Since equation (50) and the vanishing of (52) holds in the ring $\mathbb{C}\left[L^{ \pm 1}\right]\left[A_{2}, C_{1}^{-1}\right]$, we have proven the identity

$$
\frac{\partial \mathcal{F}_{h}^{\mathrm{SQ}}}{\partial T}-\langle H\rangle_{h, 1}^{\mathrm{SQ}}=0
$$

in the ring $\mathbb{C}\left[L^{ \pm 1}\right]\left[A_{2}, C_{1}^{-1}\right]$. The proof of

$$
\frac{\partial^{2} \mathcal{F}_{h}^{\mathrm{SQ}}}{\partial T^{2}}-\langle H, H\rangle_{h, 2}^{\mathrm{SQ}}=0
$$

in the ring $\mathbb{C}\left[L^{ \pm 1}\right]\left[A_{2}, C_{1}^{-1}\right]$ is identical. The proof of the lifting completes the proof of Theorem 2 .

7.4. Explicit calculations in genus 2. We present here the full calculation of $\mathcal{F}_{2}^{S Q}$ for $K \mathbb{P}^{2}$. The 7 stable graphs of genus 2 are: 


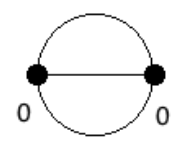

$\Gamma_{1}$

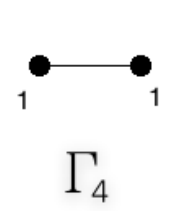

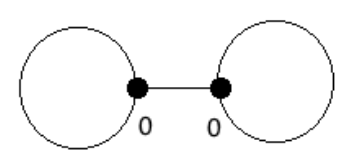

$\Gamma_{2}$

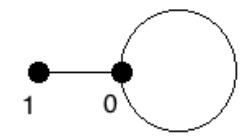

$\Gamma_{3}$
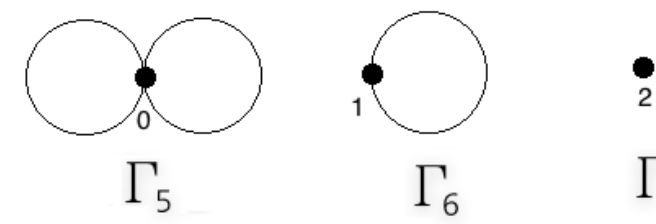

$\Gamma_{7}$

The full contribution of each stable graph $\Gamma_{i}$ is obtained by summing the contributions of all possible decorations:

$$
\begin{aligned}
\operatorname{Cont}_{\Gamma_{1}}= & \frac{24-12 L+6 L^{2}-61 L^{3}+12 L^{4}-3 L^{5}+54 L^{6}-3 L^{7}-17 L^{9}}{2592 L^{3}} \\
& +\frac{12-4 L+L^{2}-20 L^{3}+2 L^{4}+9 L^{6}}{144 L^{3}} X \\
& +\frac{6-L-5 L^{3}}{24 L^{3}} X^{2} \\
& +\frac{1}{4 L^{3}} X^{3}, \\
\operatorname{Cont}_{\Gamma_{2}}= & \frac{24-28 L+10 L^{2}-45 L^{3}+36 L^{4}-7 L^{5}+26 L^{6}-11 L^{7}-5 L^{9}}{1728 L^{3}} \\
& +\frac{36-28 L+5 L^{2}-44 L^{3}+18 L^{4}+13 L^{6}}{288 L^{3}} X \\
& +\frac{18-7 L-11 L^{3}}{48 L^{3}} X^{2} \\
& +\frac{3}{8 L^{3}} X^{3},
\end{aligned}
$$




$$
\begin{aligned}
& \operatorname{Cont}_{\Gamma_{3}}=\frac{288-190 L-25 L^{2}-364 L^{3}+145 L^{4}+74 L^{5}+97 L^{6}-25 L^{8}}{20736 L^{2}} \\
& +\frac{288-95 L-24 L^{2}-194 L^{3}+25 L^{5}}{3456 L^{2}} X \\
& +\frac{1}{8 L^{2}} X^{2} \\
& \operatorname{Cont}_{\Gamma_{4}}=\frac{2592-541 L-864 L^{2}-2229 L^{3}+720 L^{4}+897 L^{5}-575 L^{7}}{746496 L} \\
& +\frac{1}{96 L} X \\
& \operatorname{Cont}_{\Gamma_{5}}=\frac{12-8 L-11 L^{2}-8 L^{3}+5 L^{4}+16 L^{5}-L^{6}-5 L^{8}}{1728 L^{2}} \\
& +\frac{3-L-3 L^{2}-L^{3}+2 L^{5}}{72 L^{2}} X \\
& +\frac{1-L^{2}}{16 L^{2}} X^{2} \\
& \operatorname{Cont}_{\Gamma_{6}}=\frac{138+143 L-204 L^{2}-135 L^{3}-222 L^{4}+201 L^{5}+79 L^{7}}{62208 L} \\
& +\frac{23+24 L-22 L^{2}-25 L^{4}}{3456 L} X \\
& \operatorname{Cont}_{\Gamma_{7}}=\frac{281+4320 L+1785 L^{2}-2736 L^{3}-3765 L^{4}+2059 L^{6}}{3732480} .
\end{aligned}
$$

After summing the above contributions, we obtain the following result which exactly matches [2, (4.35)].

Proposition 27. The stable quotient series for $K \mathbb{P}^{2}$ in genus 2 is

$$
\begin{aligned}
\mathcal{F}_{2}^{\mathrm{SQ}}= & \frac{400-959 L^{3}+784 L^{6}-216 L^{9}}{17280 L^{3}}+\left(-\frac{1}{3}+\frac{5}{24 L^{3}}+\frac{13 L^{3}}{96}\right) X \\
& +\left(-\frac{1}{2}+\frac{5}{8 L^{3}}\right) X^{2}+\frac{5}{8 L^{3}} X^{3} .
\end{aligned}
$$

The B-model potential function for genus 2 is also calculated in [24, Section 3.5] according to graph contributions obtained from B-model physics. Though the calculation exactly matches our result in total, the individual graph contributions do not match. The relationship between the different graph contributions would be good to understand. 
7.5. Bounding the degree. The degrees in $L$ of the terms of

$$
\mathcal{F}_{g}^{\mathrm{SQ}} \in \mathbb{C}\left[L^{ \pm 1}\right]\left[A_{2}\right]
$$

for $K \mathbb{P}^{2}$ always fall in the range

$$
[9-9 g, 6 g-6] \text {. }
$$

In particular, the constant (in $A_{2}$ ) term of $\mathcal{F}_{g}^{\text {SQ }}$ missed by the holomorphic anomaly equation for $K \mathbb{P}^{2}$ is a Laurent polynomial in $L$ with degrees in the range $(53)$. The bound $(53)$ is a consequence of Proposition 20, the vertex and edge analysis of Section 6 , and the following result.

Lemma 28. The degrees in $L$ of $R_{i p}$ fall in the range

$$
[-i, 2 p] \text {. }
$$

Proof. The proof for the functions $R_{0 p}$ follows from the arguments of [33]. The proof for $R_{1 p}$ and $R_{2 p}$ follows from Lemma 9 .

For $\mathcal{F}_{2}^{\mathrm{SQ}}$, the $L$ degrees can be seen to vary between 0 and 6 in the formula of Propositon 27 when rewritten in terms of $A_{2}$ using (42). The sharper range

$$
[0,6 g-6]
$$

proposed in [2] for the $L$ degrees of $\mathcal{F}_{g}^{\mathrm{SQ}}$ is found in examples. How to derive the sharper bound from properties of the functions $R_{i p}$ is an interesting question.

\section{Holomorphic ANOMALY FOR $K \mathbb{P}^{2}$ With InSERTIONS}

8.1. Insertions. Since the stable quotient theory of $K \mathbb{P}^{2}$ has virtual dimension 0 , insertions do not play a significant role in the nonequivariant theory. However, for the equivariant stable quotient theory of $K \mathbb{P}^{2}$, insertions of all dimensions can be studied. After the specialization of torus weights

$$
\lambda_{0}=1, \quad \lambda_{1}=\zeta, \quad \lambda_{2}=\zeta^{2},
$$

in the equivariant theory (with $\zeta^{3}=1$ ), we obtain a numerical theory of $K \mathbb{P}^{2}$ with arbitrary insertions. Define the series

$$
\begin{aligned}
\mathcal{F}_{g, n}^{\mathrm{SQ}}[a, b, c] & =\left\langle\tau_{0}(1)^{a} \tau_{0}(H)^{b} \tau_{0}\left(H^{2}\right)^{c}\right\rangle_{g, n}^{\mathrm{SQ}} \\
& =\sum_{d=0}^{\infty} q^{d} \int_{\left[\bar{Q}_{g, n}\left(K \mathbb{P}^{2}, d\right)\right]^{v i r}} \prod_{i=a+1}^{a+b} \mathrm{ev}_{i}^{*}(H) \prod_{i=a+b+1}^{a+b+c} \mathrm{ev}_{i}^{*}\left(H^{2}\right),
\end{aligned}
$$

with $n=a+b+c$.

Our proof of Theorem 1 immediately yields the parallel results for the stable quotient series with insertions: 
(i) $\mathcal{F}_{g, n}^{\mathrm{SQ}}[a, b, c] \in \mathbb{C}\left[L^{ \pm 1}\right]\left[A_{2}, C_{1}, C_{1}^{-1}\right]$ for $2 g-2+n>0$,

(ii) $\mathcal{F}_{g, n}^{\mathrm{SQ}}[a, b, c]$ is of degree $\leq 3 g-3+c$ in $A_{2}$,

(iii) $\frac{\partial^{k} \mathcal{F}_{g, n}^{\mathrm{SQ}}[a, b, c]}{\partial T^{k}} \in \mathbb{C}\left[L^{ \pm 1}\right]\left[A_{2}, C_{1}, C_{1}^{-1}\right]$ for $2 g-2+n \geq 0$ and $k \geq 1$,

(iv) $\frac{\partial^{k} \mathcal{F}_{g, n}^{\mathrm{SQ}}[a, b, c]}{\partial T^{k}}$ is homogeneous of degree $k+b-c$ in $C_{1}^{-1}$.

For example, a computation by Proposition 21 yields

$$
\mathcal{F}_{0,3}^{\mathrm{SQ}}[0,0,3]=\left\langle\tau_{0}\left(H^{2}\right)^{3}\right\rangle_{g, n}^{\mathrm{SQ}}=-\frac{1}{3}\left(\frac{C_{1}}{L}\right)^{3} .
$$

A natural question is whether a holomorphic anomaly equation of the form of Theorem 2 holds for $\mathcal{F}_{g, n}^{\mathrm{SQ}}[a, b, c]$. The answer is yes, but with an additional descendent term.

Insertions of higher powers of $H$ can also be included in the stable quotient theory of $K \mathbb{P}^{2}$. However, because of the specialization of torus weights (54), insertions of $H^{k}$ for $k \geq 3$ can be reduced to insertions of $1, H, H^{2}$ via the relation

$$
\tau_{0}\left(H^{r}\right)=\tau_{0}\left(H^{s}\right) \quad \text { for } \quad r \equiv s \quad \bmod 3 .
$$

8.2. Holomorphic anomaly equation. Let $\pi$ be a morphism to the moduli space of stable curves determined by the domain,

$$
\pi: \bar{Q}_{g, n}\left(K \mathbb{P}^{2}, d\right) \rightarrow \bar{M}_{g, n}
$$

Define following the series of stable quotient invariants with descendents,

$$
\mathcal{F}_{g, n}^{\mathrm{SQ}}[a, b, c, \delta]=\left\langle\tau_{0}(1)^{a} \tau_{0}(H)^{b} \tau_{0}\left(H^{2}\right)^{c} \widetilde{\tau}_{1}(H)^{\delta}\right\rangle_{g, n=a+b+c+\delta}^{\mathrm{SQ}} .
$$

The descendent $\widetilde{\tau}_{1}(H)$ here ${ }^{19}$ corresponds to the insertion

$$
\pi^{*}\left(\psi_{i}\right) \cdot \mathrm{ev}_{i}^{*}(H)
$$

with respect to the cotangent line pulled-back via $\pi$.

Theorem 29. For $2 g-2+n>0$ and a partition $n=a+b+c$,

$$
\begin{aligned}
\frac{1}{C_{1}^{2}} \frac{\partial \mathcal{F}_{g, n}^{\mathrm{SQ}}[a, b, c]}{\partial A_{2}}= & \frac{1}{2} \sum \frac{\partial \mathcal{F}_{g_{1}, n_{1}}^{\mathrm{SQ}}\left[a_{1}, b_{1}, c_{1}\right]}{\partial T} \frac{\partial \mathcal{F}_{g_{2}, n_{2}}^{\mathrm{SQ}}\left[a_{2}, b_{2}, c_{2}\right]}{\partial T} \\
+ & \frac{1}{2} \frac{\partial^{2} \mathcal{F}_{g-1, n}^{\mathrm{SQ}}[a, b, c]}{\partial T^{2}} \\
& -\frac{1}{3} c \mathcal{F}_{g, n}^{\mathrm{SQ}}[a, b, c-1,1] .
\end{aligned}
$$

\footnotetext{
${ }^{19}$ The tilde is used to indicate the pulled-back cotangent line.
} 
The sum in the first term on the right is over all genus decompositions

$$
g_{1}+g_{2}=g
$$

and all distributions of the $n$ markings to the two parts. The point distributions determines decompositions

$$
n_{1}+n_{2}=n, \quad a_{1}+a_{2}=a, \quad b_{1}+b_{2}=b, \quad c_{1}+c_{2}=c .
$$

In fact, each such decomposition occurs

$$
\left(\begin{array}{c}
a \\
a_{1}, a_{2}
\end{array}\right)\left(\begin{array}{c}
b \\
b_{1}, b_{2}
\end{array}\right)\left(\begin{array}{c}
c \\
c_{1}, c_{2}
\end{array}\right)
$$

times in the sum. The distributions are required to satisfy

$$
2 g_{1}-2+n_{1} \geq 0, \quad 2 g_{2}-2+n_{2} \geq 0 .
$$

The unstable genus 0 cases in the sum are defined by

$$
\frac{\partial \mathcal{F}_{0,2}^{\mathrm{SQ}}\left(H^{r}, H^{s}\right)}{\partial T}=\left\langle H^{r}, H^{s}, H\right\rangle_{0,3}^{\mathrm{SQ}},
$$

and the unstable genus 1 cases are defined as in Section 7.2 .

In the second term on the right, further unstable terms in genus 0 and 1 may appear. In genus 0 , the definitions are

$$
\begin{aligned}
\frac{\partial^{2} \mathcal{F}_{0,2}^{\mathrm{SQ}}\left(H^{r}, H^{s}\right)}{\partial T^{2}} & =\left\langle H^{r}, H^{s}, H, H\right\rangle_{0,4}^{\mathrm{SQ}}, \\
\frac{\partial^{2} \mathcal{F}_{0,1}^{\mathrm{SQ}}\left(H^{r}\right)}{\partial T^{2}} & =\left\langle H^{r}, H, H\right\rangle_{0,3}^{\mathrm{SQ}} .
\end{aligned}
$$

In genus 1 , the unstable cases are defined again as in Section 7.2. Together, the first two terms on right exactly match the holomorphic anomaly equation of Theorem 2 for $K \mathbb{P}^{2}$ without insertions.

The inclusion and precise form of the new third term was motivated by the recent work of Oberdieck and Pixton [32] on the holomorphic anomaly equation for the elliptic curve $E$. While our theory of $K \mathbb{P}^{2}$ and the theory of $E$ appear to have little in common, at least two features are parallel: both targets are Calabi-Yau and both theories admit nontrivial insertions 20 . Oberdieck and Pixton prove a holomorphic anomaly equation (at the cycle level) for the elliptic curve where the differentiation on the left side is with respect to the Eisenstein series $E_{2}$ (instead of $A_{2}$ here for $K \mathbb{P}^{2}$ ). The equation of Oberdieck and Pixton has a third term exactly involving a single pulled-back descendent. ${ }^{21}$

\footnotetext{
${ }^{20}$ The moduli spaces of maps to $E$ have positive virtual dimension.

${ }^{21}$ After attending Pixton's lecture at the Institute Henri Poincaré in Paris in March 2017, we realized the parallel term is correct for our $K \mathbb{P}^{2}$ theory.
} 
8.3. Proof of Theorem 29. The localization formula of Proposition 21 can be easily extended to include the new descendent insertion.

Proposition 30. We have

$$
\begin{aligned}
& \operatorname{Cont}_{\Gamma}\left(H^{k_{1}}, \ldots, H^{k_{n}}, \pi^{*}\left(\psi_{n+1}\right) H\right)= \\
& \frac{1}{|\operatorname{Aut}(\Gamma)|} \sum_{\mathrm{A} \in \mathbb{Z}_{\geq 0}^{\mathrm{F}}} \prod_{v \in \mathrm{V}} \operatorname{Cont}_{\Gamma}^{\mathrm{A}}(v) \prod_{e \in \mathrm{E}} \operatorname{Cont}_{\Gamma}^{\mathrm{A}}(e) \prod_{l \in \mathrm{L}} \operatorname{Cont}_{\Gamma}^{\mathrm{A}}(l),
\end{aligned}
$$

where the leg contribution is

$$
\operatorname{Cont}_{\Gamma}^{\mathrm{A}}(l)=(-1)^{\mathrm{A}(l)-1}\left[e^{-\frac{\langle\langle 1,1\rangle\rangle_{0,2}^{\mathrm{p}(l), 0+}}{z}} \overline{\mathbb{S}}_{\mathrm{p}(l)}\left(H^{k_{l}}\right)\right]_{z^{\mathrm{A}(l)-1}}
$$

for $l \in\{1, \ldots, n\}$ and

$$
\operatorname{Cont}_{\Gamma}^{\mathrm{A}}(l)=(-1)^{\mathrm{A}(l)-1}\left[e^{-\frac{\langle\langle 1,1\rangle\rangle_{0,2}^{\mathrm{p}(l), 0+}}{z}} z \overline{\mathrm{S}}_{\mathrm{p}(l)}(H)\right]_{z^{\mathrm{A}(l)-1}}
$$

for $l=n+1$. The vertex and edge contributions are same as before.

Let $l$ be a leg of $\Gamma$ with insertion $H^{2}$. The $X$ derivative of the leg contribution of $l$ is

$$
\frac{\partial \operatorname{Cont}_{\Gamma}^{\mathrm{A}}(l)}{\partial X}=\left\{\begin{aligned}
(-1)^{a} \frac{L}{C_{1} C_{2}} \frac{R_{1 a-2}}{\lambda_{\mathrm{p}(l)}^{a-3}} & \text { if } a \geq 2 \\
0 & \text { if } a=1,
\end{aligned}\right.
$$

where $a=\mathrm{A}(l)$. Let $\tilde{l}$ be a leg of $\Gamma$ with insertion $\pi^{*}(\psi) H$. The $X$ derivative of the leg contribution of $\widetilde{l}$ is

$$
\operatorname{Cont}_{\Gamma}^{\mathrm{A}}(\widetilde{l})=\left\{\begin{aligned}
(-1)^{a+1} \frac{L}{C_{1}} \frac{R_{1 a-2}}{\lambda_{\mathrm{p}(\tilde{l})}^{a-3}} & \text { if } a \geq 2 \\
0 & \text { if } a=1
\end{aligned}\right.
$$

where $a=\mathrm{A}(\widetilde{l})$. Hence, when $\mathrm{p}(l)=\mathrm{p}(\widetilde{l})$ and $\mathrm{A}(l)=\mathrm{A}(\widetilde{l})$, we obtain the equation ${ }^{22}$

$$
\frac{1}{C_{1}^{2}} \frac{\partial \operatorname{Cont}_{\Gamma}^{\mathrm{A}}(l)}{\partial A_{2}}=\frac{L^{3}}{3 C_{1}^{2}} \frac{\partial \operatorname{Cont}_{\Gamma}^{\mathrm{A}}(l)}{\partial X}=-\frac{1}{3} \operatorname{Cont}_{\Gamma}^{\mathrm{A}}(\tilde{l}),
$$

which explains the third term on the right side of holomorphic anomaly equation of Theorem 29. The proof Theorem 29 then follows by exactly the same argument used for the proof Theorem 2.

In fact, the same proof yields a general holomorphic anomaly equation for all series including the insertion $\widetilde{\tau}_{1}(H)$,

\footnotetext{
${ }^{22}$ We have used the identity $L^{3}=C_{1}^{2} C_{2}$ obtained from 29.
} 


$$
\begin{aligned}
\frac{1}{C_{1}^{2}} \frac{\partial \mathcal{F}_{g, n}^{\mathrm{SQ}}[a, b, c, \delta]}{\partial A_{2}}= & \frac{1}{2} \sum \frac{\partial \mathcal{F}_{g_{1}, n_{1}}^{\mathrm{SQ}}\left[a_{1}, b_{1}, c_{1}, \delta_{1}\right]}{\partial T} \frac{\partial \mathcal{F}_{g_{2}, n_{2}}^{\mathrm{SQ}}\left[a_{2}, b_{2}, c_{2}, \delta_{2}\right]}{\partial T} \\
+ & \frac{1}{2} \frac{\partial^{2} \mathcal{F}_{g-1, n}^{\mathrm{SQ}}[a, b, c, \delta]}{\partial T^{2}} \\
& -\frac{1}{3} c \mathcal{F}_{g, n}^{\mathrm{SQ}}[a, b, c-1, \delta+1] .
\end{aligned}
$$

\section{REFERENCES}

[1] M. Aganagic, A. Klemm, M. Mariño, C. Vafa, The topological vertex, Comm. Math. Phys. 254 (2005), 425-478.

[2] M. Alim, E. Scheidegger, S.-T. Yau, J. Zhou, Special polynomial rings, quasi modular forms and duality of topological strings, Adv. Theor. Math. Phys. 18 (2014), 401-467.

[3] K. Behrend, B. Fantechi, The intrinsic normal cone, Invent. Math. 128 (1997), 45-88.

[4] M. Bershadsky, S. Cecotti, H. Ooguri, and C. Vafa, Holomorphic anomalies in topological field theories, Nucl. Phys. B405 (1993), 279-304.

[5] V. Bouchard, A. Klemm, M. Mariño, and S. Pasquetti, Remodelling the Bmodel, Comm. Math. Phys. 287 (2009), 117-178.

[6] I. Ciocan-Fontanine and B. Kim, Moduli stacks of stable toric quasimaps, Adv. in Math. 225 (2010), 3022-3051.

[7] I. Ciocan-Fontanine and B. Kim, Wall-crossing in genus zero quasimap theory and mirror maps, Algebr. Geom. 1 (2014), 400-448.

[8] I. Ciocan-Fontanine and B. Kim, Big I-functions in Development of moduli theory Kyoto 2013, 323-347, Adv. Stud. Pure Math. 69, Math. Soc. Japan, 2016.

[9] I. Ciocan-Fontanine and B. Kim, Quasimap wallcrossings and mirror symmetry, arXiv:1611.05023

[10] I. Ciocan-Fontanine and B. Kim, Higher genus quasimap wall-crossing for semi-positive targets, JEMS 19 (2017), 2051-2102.

[11] I. Ciocan-Fontanine, B. Kim, and D. Maulik, Stable quasimaps to GIT quotients, J. Geom. Phys. 75 (2014), 17-47.

[12] E. Clader, F. Janda, and Y. Ruan, Higher genus quasimap wall-crossing via localization, arXiv:1702.03427.

[13] Y. Cooper and A. Zinger, Mirror symmetry for stable quotients invariants, Michigan Math. J. 63 (2014), 571-621.

[14] K. Costello and S. Li, Quantum BCOV theory on Calabi-Yau manifolds and the higher genus B-model, arXiv:1201.4501.

[15] D. Cox and S. Katz, Mirror symmetry and algebraic geometry, Mathematical Surveys and Monographs 68: Amer. Math. Soc., Providence, RI, 1999.

[16] B. Eynard, M. Mariño, and N. Orantin, Holomorphic anomaly and matrix models, JHEP 58 (2007).

[17] B. Fang, M. C.-C. Liu, and Z. Zong, On the remodelling conjecture for toric Calabi-Yau 3-orbifolds, arXiv:1604.07123. 
[18] W. Fulton and R. Pandharipande, Notes on stable maps and quantum cohomology, Algebraic geometry - Santa Cruz 1995, 45-96, Proc. Sympos. Pure Math. 62, Part 2: Amer. Math. Soc., Providence, RI, 1997.

[19] A. Givental, Equivariant Gromov-Witten invariants, Internat. Math. Res. Notices 13 (1996), 613-663.

[20] A. Givental, Elliptic Gromov-Witten invariants and the generalized mirror conjecture, Integrable systems and algebraic geometry (Kobe/Kyoto, 1997), 107-155, World Sci. Publ., River Edge, NJ, 1998.

[21] A. Givental, Semisimple Frobenius structures at higher genus, Internat. Math. Res. Notices 23 (2001), 613-663.

[22] T. Graber, R. Pandharipande, Localization of virtual classes, Invent. Math. 135 (1999), 487-518.

[23] B. Kim and H. Lho, Mirror theorem for elliptic quasimap invariants, Geom. and Top. (to appear).

[24] A. Klemm and E. Zaslow, Local mirror symmetry at higher genus, arXiv:hepth/9906046.

[25] M. Kontsevich, Enumeration of rational curves via torus actions in The moduli space of curves (Texel Island, 1994), 335-368, Progr. Math. 129: Birkhuser Boston, Boston, MA, 1995.

[26] Y.-P. Lee and R. Pandharipande, Frobenius manifolds, Gromov-Witten theory and Virasoro constraints, https://people.math.ethz.ch/ ${ }^{\sim}$ rahul/, 2004.

[27] H. Lho and R. Pandharipande, Holomorphic anomaly equations for the formal quintic, arXiv:1803.01409.

[28] H. Lho and R. Pandharipande, Holomorphic anomaly equation for twisted theories on projective space, in preparation.

[29] A. Marian, D. Oprea, Dragos, R. Pandharipande, The moduli space of stable quotients, Geom. Topol. 15 (2011), 1651-1706.

[30] D. Maulik, A. Oblomkov, A. Okounkov, and R. Pandharipande, The GromovWitten/Donaldson-Thomas correspondence for toric 3-folds, Invent. Math. 186 (2011), 435-479.

[31] D. Maulik and R. Pandharipande, New calculations in Gromov-Witten theory, PAMQ 4 (2008), 469-500.

[32] G. Oberdieck and A. Pixton, Gromov-Witten theory of elliptic fibrations: Jacobi forms and holomorphic anomaly equations, arXiv:1709.01481.

[33] D. Zagier and A. Zinger, Some properties of hypergeometric series associated with mirror symmetry in Modular Forms and String Duality, 163-177, Fields Inst. Commun. 54, AMS 2008.

Department of Mathematics, ETH ZÜrich

E-mail address: hyenho.1ho@math.ethz.ch

Department of Mathematics, ETH ZÜRICH

E-mail address: rahul@math.ethz.ch 